See discussions, stats, and author profiles for this publication at: https://www.researchgate.net/publication/342347121

\title{
Effects of parameterization and knot placement techniques on primal and mixed isogeometric collocation formulations of spatial shear-deformable beams with varying curvature and tor...
}

Article in Computers \& Mathematics with Applications · June 2020

DOI: 10.1016/j.camwa.2020.06.006

CITATIONS

READS

0

4 authors:

Enzo Marino

University of Florence

34 PUBLICATIONS 286 CITATIONS

SEE PROFILE

Deyed Farhad Hosseini

Ferdowsi University Of Mashhad

15 PUBLICATIONS 90 CITATIONS

SEE PROFILE

A. Ali Hashemian

Basque Center for Applied Mathematics

18 PUBLICATIONS 110 CITATIONS

SEE PROFILE

Alessandro Reali

Interdisciplinary Center (IDC) Herzliya

13 PUBLICATIONS 9 CITATIONS

SEE PROFILE

Some of the authors of this publication are also working on these related projects: 


\title{
Effects of parameterization and knot placement techniques on primal and mixed isogeometric collocation formulations of spatial shear-deformable beams with varying curvature and torsion
}

\author{
Enzo Marino ${ }^{\mathrm{a}, *}$, Seyed Farhad Hosseini ${ }^{\mathrm{b}}$, Ali Hashemian ${ }^{\mathrm{c}, \mathrm{d}}$, Alessandro Reali \\ ${ }^{a}$ Department of Civil and Environmental Engineering, University of Florence, Firenze, Italy \\ ${ }^{b}$ Sun-Air Research Institute, Ferdowsi University of Mashhad, Mashhad, Iran \\ ${ }^{c}$ BCAM - Basque Center for Applied Mathematics, Bilbao, Basque Country, Spain \\ ${ }^{d}$ Department of Mechanical Engineering, Hakim Sabzevari University, Sabzevar, Iran \\ ${ }^{e}$ Department of Civil Engineering and Architecture, University of Pavia, Pavia, Italy
}

\section{Abstract}

We present a displacement-based and a mixed isogeometric collocation (IGA-C) formulation for free-form, three-dimensional, shear-deformable beams with high and rapidly-varying curvature and torsion. When such complex shapes are concerned, the approach used to build the IGA geometric model becomes relevant. Although IGA-C has been so far successfully applied to a wide range of problems, the effects that different parameterization and knot placement techniques may have on the accuracy of collocation-based formulations is still an unexplored field. To fill this gap, primal and mixed formulations are used combining two parameterization methods (chord-length and equally spaced) with two knot placement techniques (uniformly spaced and De Boor). With respect to the space-varying Frenet local frame, we derive the strong form of the governing equations in a compact form through the definition of two matrix operators conveniently used to perform first and second order derivatives of the vector fields involved in the formulations. This approach is very efficient and easy to implement within a collocation-based scheme. Several challenging numerical experiments allow to test the different considered parameterizations and knot placement techniques, revealing in particular that with the primal formulation an equally spaced parameterization is definitively the most recommended choice and it should always be used with an approximation degree of, at least, $p=6$, although some caution must be adopted when very high

\footnotetext{
${ }^{*}$ Corresponding author

Email address: enzo.marino@unifi.it +39 0552758858 (Enzo Marino)
} 
Jacobians and small curvatures occur. The same holds for the mixed formulation, with the difference that $p=4$ is enough to yield accurate results.

Keywords: Isogeometric collocation, shear-deformable free-form beams, primal and mixed beam formulations, parameterization and knot placement

1

\section{Introduction}

The isogeometric collocation (IGA-C) method was proposed in [1] with the aim of combining the attributes of isogeometric analysis (IGA) [2] with the low computational cost of collocation. The primary goal of IGA is to represent accurately the model geometry even with extremely coarse discretizations. Moreover, in contrast to standard finite element analysis (FEA), in IGA mesh refinement is significantly simplified since there is no need for communication with the Computer Aided Design (CAD) model once the initial mesh is constructed. IGA makes use of functions commonly adopted in CAD, such as B-splines and NURBS [3], both for the geometry representation and the spatial discretization of the differential equations. The use of such basis functions, characterized by high and adjustable smoothness, has proven to achieve increased accuracy and robustness on a per degree-offreedom basis compared with standard FEA [4-7]. An exhaustive presentation of IGA is found in [8]. The application of IGA is growing fast in many branches of science and engineering, such as, e.g., solid mechanics [9-13], fluid mechanics [14-16], electromagnetics $[17,18]$, and eigenvalue problems $[19,20]$.

A side-effect of using high-order basis functions is the fast growth of the computational cost due to the larger number of quadrature points. Moreover, the high smoothness degree that B-splines or NURBS typically possess across the elements makes Gauss integration rules suboptimal $[21,22]$. The development of more efficient integration schemes is currently an open problem, although significant progress has been made in [23-28]. IGA-C represents an interesting solution for this problem since the need for numerical quadrature is completely removed due to the discretization of the strong form of the governing equations. IGA-C requires only one evaluation point per degree of freedom, regardless of the approximation degree, resulting in a much faster method compared to standard Galerkin-based IGA based on Gauss quadrature [29]. 
IGA-C has been successfully applied to linear problems [1, 29, 30], phase-field modeling of immiscible fluids [31] and ferroelastic materials [32], contact problems [33, 34], and hyperelasticity [34]. New connections between Galerkin and collocation methods were established in [35]. Timoshenko beam formulations were proposed in [36-40]. Bernoulli-Euler beams and Kirchhoff plates were addressed in [41], and Reissner-Mindlin plate and shell problems in [42] and [43], respectively. Kirchhoff-Love plate and shell problems were studied in [44]. Laminated composite plates have been recently addressed in [45]. Nonlinear planar Kirchhoff rods were formulated in [46]. In linear dynamics, an explicit IGA-C formulation was introduced in [30] and more recently an explicit higher-order space- and time-accurate method for elastodynamics was proposed in [47]. In [48-50] IGA-C was extended to the static problem of geometrically nonlinear three-dimensional shear-deformable beams, whereas the method was extended to the dynamic problem using an implicit quaternion-based formulation in [51], an explicit formulation based on the spatial incremental rotation vector in [52], and an implicit formulation based on the material incremental rotation vector in [53].

The simulations of highly curved three-dimensional rods involves the concept of "analysisaware modeling", firstly proposed by Cohen et al. [54], which is aimed at constructing geometries suitable for isogeometric analysis. In some other researches related to this topic, $\mathrm{Xu}$ et al. $[55,56]$ employed the optimization methods to rearrange the position of middle control points in $2 \mathrm{D}$ and $3 \mathrm{D}$ cases to reach a better parameterization for computational domains. Casquero et al. [57] employed analysis suitable T-splines for solving second and fourth order boundary value problems using the isogeometric collocation method. The effect of perturbing control points in different computational domains have been investigated by Lipton et al. [58]. They showed that changing the position of middle control points will affect the parameterization and therefore IGA results while keeping the geometry visually unchanged.

Free-form curved beam geometries with any desired shape can be generated for isogeometric analysis in two ways - by direct input from a CAD environment (e.g., Rhino) or by fitting a curve to a set of data points (obtained by, e.g., an implicit algebraic equation or a point cloud). In the first case, all spline geometry information such as the position of control points and the knot vector are imported from the CAD system. In this regard, a practical 
method in order to modify the geometry in accordance with IGA requirements (while keeping the exact shape) is the curve reparameterization technique presented by Hosseini et al. [59]. Curve reparameterization can change the (probably) unsuitable initial parameterization of the imported geometry modifying its Jacobian. In the case that the beam geometry is given by a series of input data points, generally a curve approximation is used to construct the required IGA suitable geometry. Two main steps may be identified in a general B-spline fitting process, namely, parameter selection and knot vector generation [3]. Parameterization directly affects the geometric factors related to derivatives (such as the Jacobian). On the other hand, the knot sequence determines the position of nodes in the physical geometry and collocation points. Therefore, an inappropriate combination of parameterization and knot placement methods directly influences the accuracy of IGA-C (and, more in general, IGA) results. These issues are also the topics of interest in other engineering applications, e.g., trajectory planning in robotics $[60,61]$ and machining processes $[62,63]$. The importance of parameterization in IGA is studied in different researches. For example, Kolman et al. [64] investigated the effect of nonlinear and linear parameterizations obtained by uniformly-spaced control points and Greville abscissa formula, respectively. The comparison between typical parameter selection strategies (namely uniformly-spaced, chord-length, and centripetal parameterizations) in constructing free-form curved beam structures are addressed by Hosseini et al. [65], which show the effectiveness of chord-length parameterization when non-uniform input data points are given. Parameterization is also briefly discussed in other researches such as [66-69]. Very recently, the effect of knot placement techniques in IGA of free-form Euler-Bernoulli curved beams is investigated in [70] where the superiority of De Boor knot placement technique is shown.

While the concept of analysis-aware modeling has already received attentions and some key results have been obtained in the Galerkin-based IGA, to the best of our knowledge, there is no existing study addressing the effects that parameterization and knot placement techniques have on the accuracy of collocation-based formulations. Moreover, in all the above mentioned papers on collocation, the problem of spatial rods with varying curvature and torsion has not been deeply investigated. Therefore, in this paper we present primal and mixed IGA-C formulations for rods with strongly varying curvature and torsion and 
systematically discuss the effects that different combinations of parameterization and knot placement techniques have on the accuracy of the methods. The main objective of the current research is to contribute to the development of efficient analysis-aware modeling of structures with complex free-form geometry.

The remainder of this paper is organized as follows. Section 2 reviews the notations of differential geometry of 3D curves in space, followed by the governing equations of spatial free-form curved beams in Section 3. In Section 4, the definition of B-spline curves (including the curve approximation procedure) is presented. The different parameter selection and knot vector generation approaches are presented in this section as well. Then, in Section 5, the displacement-based and mixed formulations of isogeometric collocation are introduced and in Section 6, different case studies and numerical examples are presented. Finally, Section 7 draws the conclusions of this work.

\section{Brief review of differential geometry of spatial curves}

Let $s \mapsto \boldsymbol{c}(s) \in \mathbb{R}^{3}$, with $s \in I_{s}=[0, L] \subset \mathbb{R}$, be a smooth curve parameterized by the arc length $s$. The Frenet frame $\{\boldsymbol{t}, \boldsymbol{n}, \boldsymbol{b}\}$ is defined as follows [71]

$$
\begin{aligned}
\boldsymbol{t} & =\boldsymbol{c},_{s}, \\
\boldsymbol{n} & =\frac{\boldsymbol{c},_{s s}}{\left\|\boldsymbol{c},_{s s}\right\|}, \\
\boldsymbol{b} & =\boldsymbol{t} \times \boldsymbol{n},
\end{aligned}
$$

where with $(\cdot)_{s}$ we indicate the partial derivative with respect to the coordinate $s$. In the above equations, $\boldsymbol{t}$ is the unit length tangent vector to the curve at $s, \boldsymbol{n}$ is the unit length normal vector at $s$, and $\boldsymbol{b}$ is the unit length binormal vector at $s$ (see Figure 1). It is noted that $\boldsymbol{c}_{s s} \cdot \boldsymbol{c},_{s}=0$, thus $\{\boldsymbol{t}, \boldsymbol{n}, \boldsymbol{b}\}$ represents an orthonormal basis which is used to formulate the classical problem of three-dimensional shear-deformable curved rods.

The curvature $\kappa$ and torsion $\tau$ of the curve $\boldsymbol{c}$ at $s$ are defined as follows

$$
\begin{aligned}
\kappa & =\left\|\boldsymbol{c},_{s s}\right\|, \\
\tau & =-\frac{\boldsymbol{c},,_{s s}}{\kappa^{2}} \cdot\left(\boldsymbol{c}_{s} \times \boldsymbol{c},,_{s s}\right) .
\end{aligned}
$$




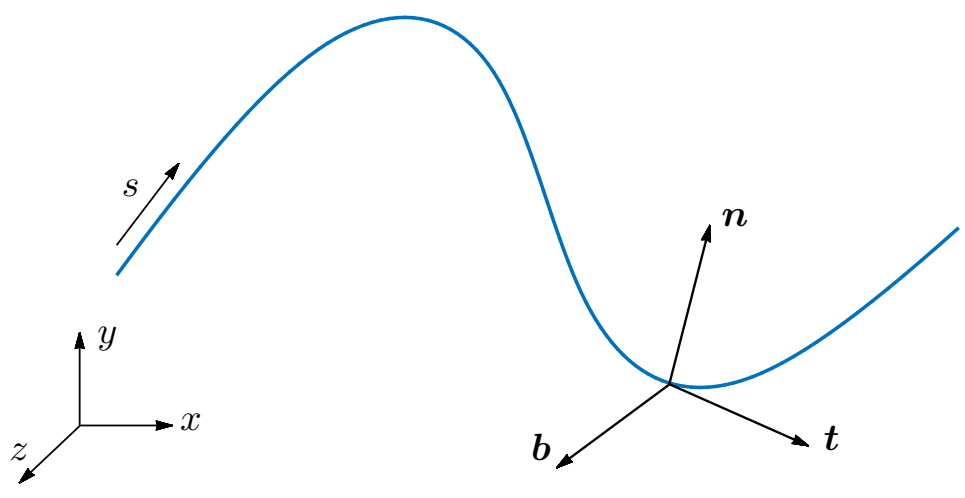

Figure 1: The orthonormal Frenet frame on a spatial free-form curved beam.

Classical Galerkin-based formulations of curved spatial rods (see, e.g., [72-75]) require only curvature and torsion as defined in Eqs. (4) and (5). In the present context, since we are concerned with the discretization of the strong form of the differential equations, the derivatives of curvature $\kappa^{\prime}$ and torsion $\tau^{\prime}$ are also needed (see details in Section 3). These derivatives are given as follows

$$
\begin{aligned}
& \kappa^{\prime}=\frac{\boldsymbol{c}, s s \cdot \boldsymbol{c},{ }_{s s s}}{\kappa}, \\
& \tau^{\prime}=\left(-\frac{\boldsymbol{c}, s_{s s}}{\kappa^{2}}+2 \frac{\boldsymbol{c}_{s s} \kappa, s}{\kappa^{3}}\right) \cdot\left(\boldsymbol{c},_{s} \times \boldsymbol{c},_{s s s}\right)-\frac{\boldsymbol{c}_{s s}}{\kappa^{2}} \cdot\left(\boldsymbol{c}_{, s s} \times \boldsymbol{c}_{s s s}+\boldsymbol{c}_{s} \times \boldsymbol{c}_{s s s s}\right) .
\end{aligned}
$$

Note that here and in the following with $(\cdot)^{\prime}$ we denote the derivative with respect to $s$ of matrix (or vector) components only.

To avoid the presentation of the governing equations (see Section 3) in components, which would be lengthy and less efficient for the following numerical formulations (see Section 5), we rearrange curvature and torsion, as well as their derivatives, in a matrix form. To this end, we rename the Frenet frame as $\left\{\mathbf{t}_{1}, \mathbf{t}_{2}, \mathbf{t}_{3}\right\}=\{\boldsymbol{t}, \boldsymbol{n}, \boldsymbol{b}\}$. The Frenet-Serret formula [71] leads to

$$
\mathbf{t}_{i, s}=\tilde{\kappa}_{i j} \mathbf{t}_{j} \text { for } i=1,2,3,
$$

where $\tilde{\kappa}_{i j}$ are the components of a skew-symmetric matrix defined as follows

$$
\widetilde{\boldsymbol{\kappa}}=\left[\begin{array}{ccc}
0 & \kappa & 0 \\
-\kappa & 0 & \tau \\
0 & -\tau & 0
\end{array}\right] \text {. }
$$

Note that in the present work a repeated index implies the summation over that index. 
In a similar way we define the matrix $\widetilde{\boldsymbol{\kappa}}^{\prime}$ as follows

$$
\widetilde{\boldsymbol{\kappa}}^{\prime}=\left[\begin{array}{ccc}
0 & \kappa^{\prime} & 0 \\
-\kappa^{\prime} & 0 & \tau^{\prime} \\
0 & -\tau^{\prime} & 0
\end{array}\right]
$$

where $\kappa^{\prime}$ and $\tau^{\prime}$ are obtained through Eqs. (6) and (7).

We remark that the Frenet frame is not the only possible choice. Other approaches employing rotation-minimizing frames, such as the Bishop frame [76, 77], would be possible. In this paper we stick to the most widely used approach, leaving the investigation of other frames to future studies.

Let $s \mapsto \boldsymbol{r}(s) \in \mathbb{R}^{3}$ be a generic vector field which, in the local basis $\left\{\mathbf{t}_{1}, \mathbf{t}_{2}, \mathbf{t}_{3}\right\}$, reads as $\boldsymbol{r}=r_{i} \mathbf{t}_{i}$. The spatial derivative of $\boldsymbol{r}$ is given by

$$
\boldsymbol{r},_{s}=r_{i, s} \mathbf{t}_{i}+r_{i} \mathbf{t}_{i, s}=r_{i, s} \mathbf{t}_{i}+\tilde{\kappa}_{i j} r_{i} \mathbf{t}_{j}=\left(r_{j, s}+\tilde{\kappa}_{i j} r_{i}\right) \mathbf{t}_{j}=\boldsymbol{r}^{\prime}+\widetilde{\boldsymbol{\kappa}}^{\top} \boldsymbol{r}=\boldsymbol{r}^{\prime}-\widetilde{\boldsymbol{\kappa}} \boldsymbol{r}=\boldsymbol{r}^{\prime}-\boldsymbol{\kappa} \times \boldsymbol{r},
$$

where Eq. (8) and the skew-symmetry of $\widetilde{\boldsymbol{\kappa}}$ have been exploited. We have also defined the axial vector of $\widetilde{\boldsymbol{\kappa}}$ as $\boldsymbol{\kappa}=-[\tau, 0, \kappa]^{\top 1}$.

\section{Governing equations in strong form}

We start this section by recalling the strong form of the balance equations which, for any $s \in(0, L)$, are given as follows

$$
\begin{array}{r}
\mathbf{n},_{s}+\overline{\mathbf{n}}=\mathbf{0}, \\
\mathbf{m},_{s}+\mathbf{t}_{1} \times \mathbf{n}+\overline{\mathbf{m}}=\mathbf{0},
\end{array}
$$

where we have used $\mathbf{t}_{1}=\boldsymbol{t}=\boldsymbol{c},{ }_{s}$. In the above equation, $\mathbf{n}$ and $\mathbf{m}$ are the internal forces and moment vectors, respectively (see Figure 2); and $\overline{\mathbf{n}}$ and $\overline{\mathbf{m}}$ are the distributed external force and moment vectors, respectively.

\footnotetext{
${ }^{1}$ With the symbol $\sim$ we denote elements of so(3), that is the set of $3 \times 3$ skew-symmetric matrices. Furthermore, for any skew-symmetric matrix $\widetilde{\boldsymbol{a}} \in \operatorname{so}(3), \boldsymbol{a}=\operatorname{axial}(\widetilde{\boldsymbol{a}})$ indicates the axial vector of $\widetilde{\boldsymbol{a}}$ such that $\widetilde{\boldsymbol{a}} \boldsymbol{h}=\boldsymbol{a} \times \boldsymbol{h}$, for any $\boldsymbol{h} \in \mathbb{R}^{3}$.
} 
(a) Displacement and rotation fields.

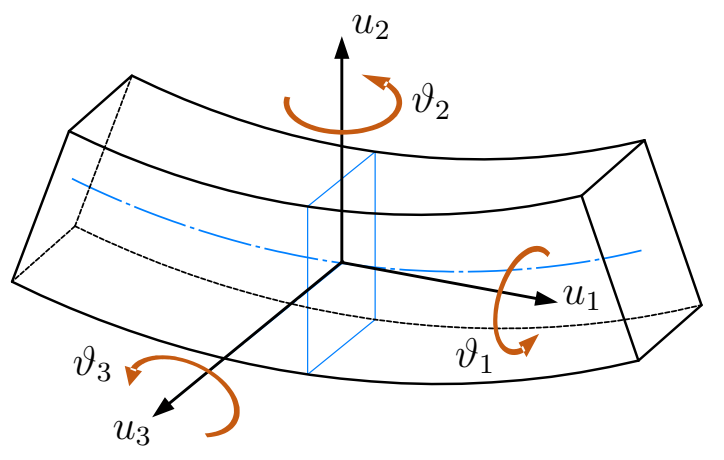

According to the fundamental assumption for the shear-deformable beam model, the beam kinematics is completely described by two vector fields $\boldsymbol{u}$ and $\boldsymbol{\vartheta}$ expressing the displacement of the centroid line of the beam and the rotation of the cross section at each point of the centroid line of the beam, respectively (see Figure 2). The components of the kinematic fields are given with respect to the local basis, namely $\boldsymbol{u}=u_{i} \mathbf{t}_{i}$ and $\boldsymbol{\vartheta}=\vartheta_{i} \mathbf{t}_{i}$.

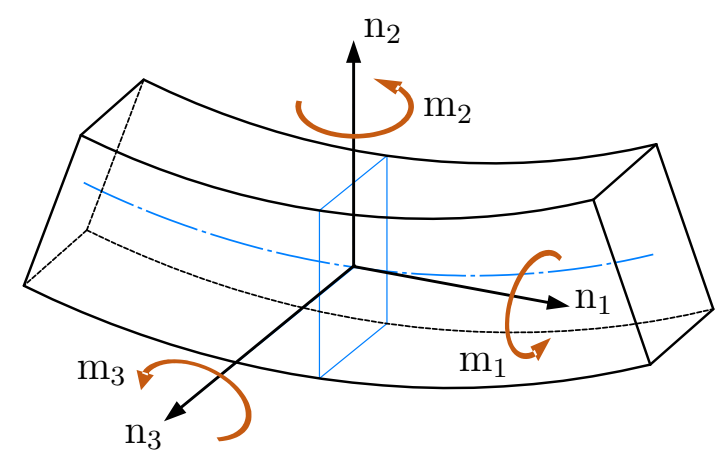

(b) Internal forces and couples.

Figure 2: Components in the local frame $\left\{\mathbf{t}_{1}, \mathbf{t}_{2}, \mathbf{t}_{3}\right\}$ of (a) displacement $\boldsymbol{u}$ and rotation $\boldsymbol{\vartheta}$ fields and (b) internal force $\mathbf{n}$ and moment $\mathbf{m}$.

The strain measures are defined as follows $[72,73]$

$$
\begin{aligned}
\boldsymbol{\varepsilon} & =\boldsymbol{u},_{s}+\mathbf{t}_{1} \times \boldsymbol{\vartheta}, \\
\chi & =\boldsymbol{\vartheta},_{s},
\end{aligned}
$$

where $\varepsilon$ is the vector of axial and shear strains, and $\chi$ is the vector of bending and torsional strains. Under the assumption of an isotropic, homogeneous, linear elastic material, the constitutive equations are given as follows

$$
\begin{aligned}
\mathbf{n} & =\mathbb{C} \varepsilon \\
\mathbf{m} & =\mathbb{D} \boldsymbol{\chi}
\end{aligned}
$$

where $\mathbb{C}=\operatorname{diag}\left(E A, G A_{2}, G A_{3}\right)$ and $\mathbb{D}=\operatorname{diag}\left(G J, E J_{2}, E J_{3}\right)$. Herein, $G A_{2}$ and $G A_{3}$ are the shear stiffnesses along the cross section principal axes, $E A$ is the axial stiffness, $G J$ is the torsional stiffness, and $E J_{2}$ and $E J_{3}$ are the principal bending stiffnesses. Note that since the strain measures and the elastic matrices $\mathbb{C}$ and $\mathbb{D}$ are expressed in the local frame, 


\subsection{Mixed formulation in strong form}

For the mixed formulation we follow the approach used in [50], where internal forces $\mathbf{n}$ and couples $\mathbf{m}$ are both considered as two additional independent variables. The system

where $\overline{\mathbf{n}}_{c}$ and $\overline{\mathbf{m}}_{c}$ are the external concentrated force and moment vectors, respectively. Dirichlet boundary conditions at $s \in\{0, L\}$ are, instead, given as

$$
\begin{aligned}
& \boldsymbol{u}=\overline{\boldsymbol{u}}_{c}, \\
& \boldsymbol{\vartheta}=\overline{\boldsymbol{\vartheta}}_{c},
\end{aligned}
$$

where $\overline{\boldsymbol{u}}_{c}$ and $\overline{\boldsymbol{\vartheta}}_{c}$ are the translation and rotation vectors expressing the prescribed kinematic conditions.

\subsection{Displacement-based formulation in strong form}

By using the derivation rule given in Eq. (11) and the constitutive equations (16) and (17), the governing equations (12) and (13) can be expressed in terms of the two independent kinematic fields $\boldsymbol{u}$ and $\boldsymbol{\vartheta}$ as follows

$$
\begin{array}{r}
\mathbb{C} \widetilde{\mathbf{t}}_{1} \boldsymbol{\vartheta}^{\prime}-\widetilde{\boldsymbol{\kappa}} \mathbb{C}_{\mathbf{t}_{1}} \boldsymbol{\vartheta}+\mathbb{C} \boldsymbol{u}^{\prime \prime}-(\widetilde{\boldsymbol{\kappa}} \mathbb{C}+\mathbb{C} \widetilde{\boldsymbol{\kappa}}) \boldsymbol{u}^{\prime}-\left(\mathbb{C} \widetilde{\boldsymbol{\kappa}}^{\prime}-\widetilde{\boldsymbol{\kappa}} \mathbb{C} \widetilde{\boldsymbol{\kappa}}\right) \boldsymbol{u}+\overline{\mathbf{n}}=0, \\
\mathbb{D} \boldsymbol{\vartheta}^{\prime \prime}-(\mathbb{D} \widetilde{\boldsymbol{\kappa}}+\widetilde{\boldsymbol{\kappa}} \mathbb{D}) \boldsymbol{\vartheta}^{\prime}+\left(\widetilde{\boldsymbol{\kappa}} \mathbb{D} \widetilde{\boldsymbol{\kappa}}-\mathbb{D} \widetilde{\boldsymbol{\kappa}}^{\prime}+\widetilde{\mathbf{t}}_{1} \mathbb{C} \widetilde{\mathbf{t}}_{1}\right) \boldsymbol{\vartheta}+\widetilde{\mathbf{t}}_{1} \mathbb{C} \boldsymbol{u}^{\prime}-\widetilde{\mathbf{t}}_{1} \mathbb{C} \widetilde{\boldsymbol{\kappa}} \boldsymbol{u}+\overline{\mathbf{m}}=0 .
\end{array}
$$

In a similar way, the Neumann boundary conditions given in Eqs. (18) and (19) can be expressed as

$$
\begin{aligned}
\mathbb{C}\left(\boldsymbol{u}^{\prime}-\widetilde{\boldsymbol{\kappa}} \boldsymbol{u}+\widetilde{\mathbf{t}}_{1} \boldsymbol{\vartheta}\right) & =\overline{\mathbf{n}}_{c}, \\
\mathbb{D}\left(\boldsymbol{\vartheta}^{\prime}-\widetilde{\boldsymbol{\kappa}} \boldsymbol{\vartheta}\right) & =\overline{\mathbf{m}}_{c} .
\end{aligned}
$$


of differential equations is obtained by coupling Eqs. (12) and (13) with the constitutive equations (16) and (17), which, by using the strain measures Eqs. (14) and (15), lead to the following system

$$
\begin{aligned}
& \mathbf{n}^{\prime}-\widetilde{\boldsymbol{\kappa}} \mathbf{n}+\overline{\mathbf{n}}=\mathbf{0}, \\
& \mathbf{m}^{\prime}-\widetilde{\boldsymbol{\kappa}} \mathbf{m}+\widetilde{\mathbf{t}}_{1} \mathbf{n}+\overline{\mathbf{m}}=\mathbf{0}, \\
& \mathbb{C} \boldsymbol{u}^{\prime}-\mathbb{C} \widetilde{\boldsymbol{\kappa}} \boldsymbol{u}+\mathbb{C} \widetilde{\mathbf{t}}_{1} \boldsymbol{\vartheta}-\mathbf{n}=\mathbf{0}, \\
& \mathbb{D} \vartheta^{\prime}-\mathbb{D} \widetilde{\boldsymbol{\kappa} \vartheta}-\mathbf{m}=\mathbf{0},
\end{aligned}
$$

where the differentiation rule given in Eq. (11) has been used. In the present mixed differential problem we have $\boldsymbol{\vartheta}, \boldsymbol{u}, \mathbf{m}, \mathbf{n}$ as unknown fields. Neumann boundary conditions valid in $s \in\{0, L\}$ are

$$
\begin{aligned}
\mathbf{n}-\overline{\mathbf{n}}_{c} & =\mathbf{0}, \\
\mathbf{m}-\overline{\mathbf{m}}_{c} & =\mathbf{0}, \\
\mathbb{C} \boldsymbol{u}^{\prime}-\mathbb{C} \widetilde{\boldsymbol{\kappa}} \boldsymbol{u}+\mathbb{C} \widetilde{\mathbf{t}_{1}} \boldsymbol{\vartheta}-\mathbf{n} & =\mathbf{0}, \\
\mathbb{D} \boldsymbol{\vartheta}^{\prime}-\mathbb{D} \widetilde{\boldsymbol{\kappa} \vartheta}-\mathbf{m} & =\mathbf{0},
\end{aligned}
$$

while Dirichlet boundary conditions are

$$
\begin{aligned}
& \boldsymbol{u}-\overline{\boldsymbol{u}}_{c}=\mathbf{0}, \\
& \boldsymbol{\vartheta}-\overline{\boldsymbol{\vartheta}}_{c}=\mathbf{0}, \\
& \mathbb{C} \boldsymbol{u}^{\prime}-\mathbb{C} \widetilde{\boldsymbol{\kappa}} \boldsymbol{u}+\mathbb{C} \widetilde{\mathbf{t}}_{1} \boldsymbol{\vartheta}-\mathbf{n}=\mathbf{0}, \\
& \mathbb{D} \boldsymbol{\vartheta}^{\prime}-\mathbb{D} \widetilde{\boldsymbol{\kappa} \vartheta}-\mathbf{m}=\mathbf{0} .
\end{aligned}
$$

\section{Geometry construction by B-spline curves}

\subsection{Definition of B-spline curves}

Following the IGA paradigm, herein, B-splines are employed for both representing the beam geometry and expressing the solution fields. Let $I_{u}=[0,1]$ be the normalized univariate domain of the spline space, a B-spline curve $u \mapsto \boldsymbol{c}(u) \in \mathbb{R}^{3}$ of degree $p$ with $n+1$ control 

single multiplicities of internal knots.
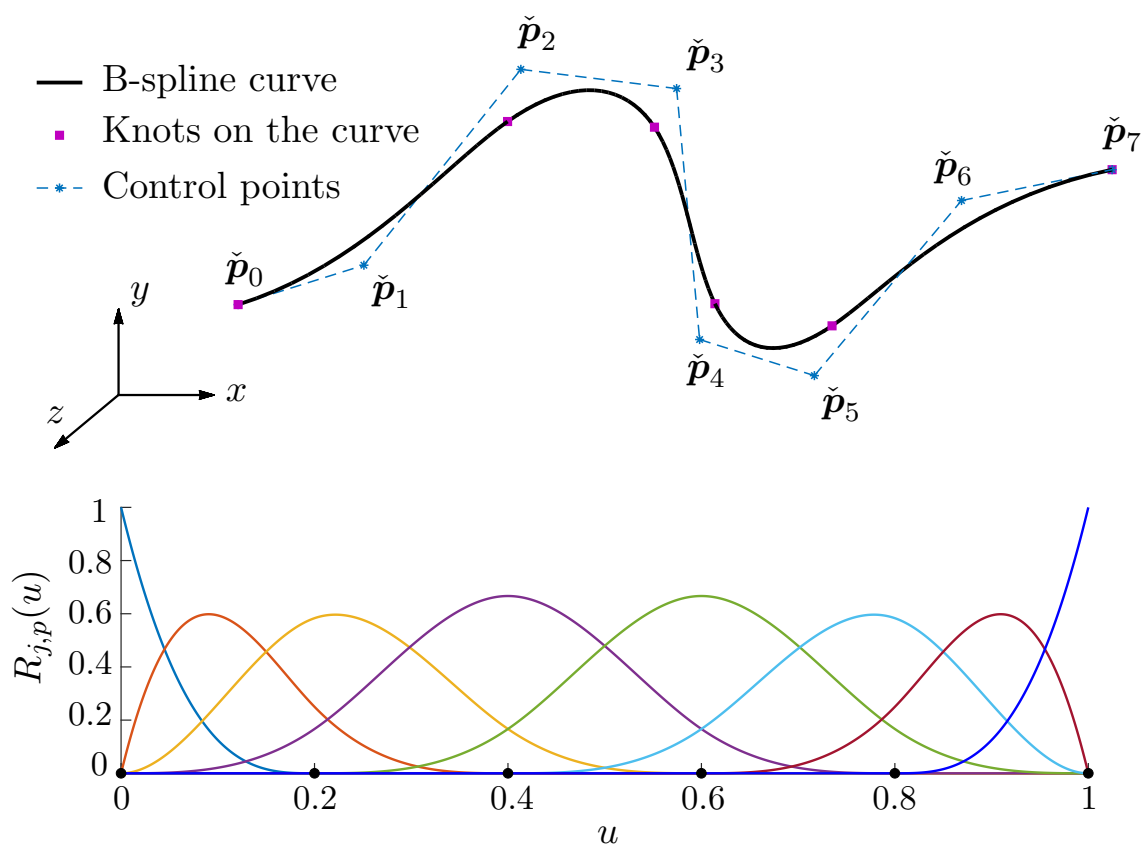

Figure 3: Top: a cubic B-spline curve in 3D space with eight control points. Bottom: cubic basis functions and respective knots on the knot vector. 


\subsection{B-spline curve fitting: parameterization and knot placement}

If a set of $h+1$ data points $\boldsymbol{d}_{0}, \boldsymbol{d}_{1}, \ldots, \boldsymbol{d}_{h}$ representing the beam geometry is available (obtained by, e.g., an algebraic equation or a point cloud), the B-spline expression of the beam can be found by a data fitting technique that is generally performed by a curve approximation (there are also other alternatives like, e.g., interpolation, mixed interpolation/approximation, and optimization-based fitting $[60,78,79])$.

Focusing on curve approximation in this paper, the curved beam geometry is to be constructed in such a way that the control points $\check{\boldsymbol{p}}_{j}$ are the output of a global curve fitting problem. In order for the geometry to be appropriately approximated by a B-spline curve, the first step is to associate the parameter $\bar{u}_{k}$ to the $k$-th data point $\boldsymbol{d}_{k}$ by applying the equally spaced or chord-length parameterization schemes [3] described, respectively, by

$$
\begin{gathered}
\bar{u}_{k}=\frac{k}{h} \quad(k=0,1, \ldots, h), \\
\bar{u}_{0}=0, \quad \bar{u}_{k}=\frac{\sum_{i=1}^{k}\left\|\boldsymbol{d}_{i}-\boldsymbol{d}_{i-1}\right\|}{\sum_{i=1}^{h}\left\|\boldsymbol{d}_{i}-\boldsymbol{d}_{i-1}\right\|} \quad(k=1,2, \ldots, h),
\end{gathered}
$$

where $\|\cdot\|$ indicates the Euclidean norm. It is remarked that in an earlier phase of our investigation, the centripetal parameterization scheme [3] was also considered. However, the results were never of particular relevance with respect to the other parameterizations.

In the next step, an appropriate knot vector should be generated to characterize the spline space of the geometry. Considering that collocation points in the IGA-C framework are normally directly obtained by the knot values, the constructed geometry will affect the solution output as well. There are different knot vector generation methods for curve/surface approximation in the literature (see, e.g., [80-82]) and the two most used techniques, namely uniform and De Boor knot placement algorithms, are presented in the following. Referring to the knot sequence of Eq. (39), in the uniform knot placement technique, which is the simplest and typical knot sequence generation algorithm in geometry construction, the internal knots are equally spaced in $I_{u}$ as

$$
u_{p+i}=\frac{i}{n-p+1}, \quad(i=1,2, \ldots, n-p) .
$$

On the other hand, in the De Boor's algorithm, which generally yields a stable and appropriate curve fitting, every knot span is guaranteed to contain at least one parameter 
$\bar{u}_{k}$. For this purpose, the internal knots should be defined as follows [3]

$$
u_{p+i}=(1-\alpha) \bar{u}_{m-1}+\alpha \bar{u}_{m}, \quad(i=1,2, \ldots, n-p)
$$

where, by defining int $(\cdot)$ as the floor function, the values of $\alpha$ and $m$ can be found as

$$
\begin{aligned}
\alpha & =i \cdot d-1, \quad(i=1,2, \ldots, n-p), \\
m & =\operatorname{int}(i \cdot d), \\
d & =\frac{h+1}{n-p+1} .
\end{aligned}
$$

Finally, the input data points can be approximated by a B-spline curve with $n+1$ control points $(n \leq h)$ where the first and last control points are simply determined as $\check{\boldsymbol{p}}_{0}=\boldsymbol{d}_{0}$ and $\check{\boldsymbol{p}}_{n}=\boldsymbol{d}_{h}$. The remaining control points $\check{\boldsymbol{P}}=\left[\check{\boldsymbol{p}}_{1}, \check{\boldsymbol{p}}_{2}, \ldots, \check{\boldsymbol{p}}_{n-1}\right]^{\top}$ are to be computed in the least-squares sense through the minimization of the following fitting function

$$
f=\sum_{k=1}^{h-1}\left\|\boldsymbol{d}_{k}-\boldsymbol{c}\left(\bar{u}_{k}\right)\right\|^{2} .
$$

In this case, the number of control points is to be determined such that a desirable fitting error and/or accuracy in the IGA-C results are achieved. By setting the derivatives $\partial f / \partial \check{\boldsymbol{p}}_{j}$ equal to zero, and employing standard matrix algebra, one obtains the control points as [3]

$$
\check{\boldsymbol{P}}=\left(\boldsymbol{B}^{\top} \boldsymbol{B}\right)^{-1} \boldsymbol{B}^{\top} \boldsymbol{Q}
$$

where $\boldsymbol{B}$ is the matrix of the basis functions at parameter values

$$
\boldsymbol{B}=\left[\begin{array}{cccc}
R_{1, p}\left(\bar{u}_{1}\right) & R_{2, p}\left(\bar{u}_{1}\right) & \ldots & R_{n-1, p}\left(\bar{u}_{1}\right) \\
R_{1, p}\left(\bar{u}_{2}\right) & R_{2, p}\left(\bar{u}_{2}\right) & \ldots & R_{n-1, p}\left(\bar{u}_{2}\right) \\
\vdots & \vdots & \ddots & \vdots \\
R_{1, p}\left(\bar{u}_{h-1}\right) & R_{2, p}\left(\bar{u}_{h-1}\right) & \ldots & R_{n-1, p}\left(\bar{u}_{h-1}\right)
\end{array}\right]
$$

and $\boldsymbol{Q}=\left[\boldsymbol{q}_{1}, \boldsymbol{q}_{2}, \ldots \boldsymbol{q}_{h-1}\right]^{\top}$ with

$$
\boldsymbol{q}_{k}=\boldsymbol{d}_{k}-R_{0, p}\left(\bar{u}_{k}\right) \boldsymbol{d}_{0}-R_{n, p}\left(\bar{u}_{k}\right) \boldsymbol{d}_{h}
$$

By employing different combinations of parameterizations (Eqs. (41) and (42)) and knot placements (Eqs. (43) and (44)), different curve fits can be obtained for a set of input data 
points and, therefore, different IGA-C results are achieved. Figure 4 illustrates the effect of different parameterizations and knot placement techniques on fitting a cubic curve to a planar dataset noting that the quality of the fitted curve would increase by employing more control points. The figure shows that in addition to the quality of the fitting process, the positions of control points and the values of their respective basis functions also depend on the combination adopted for the geometry construction.
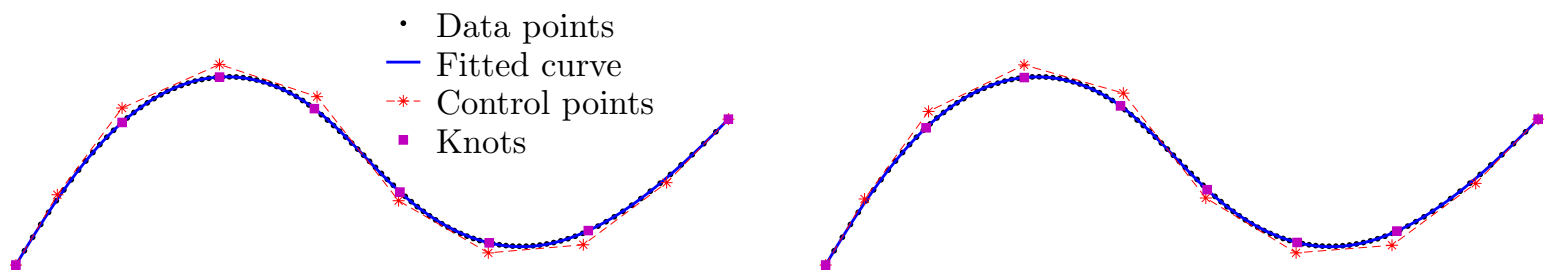

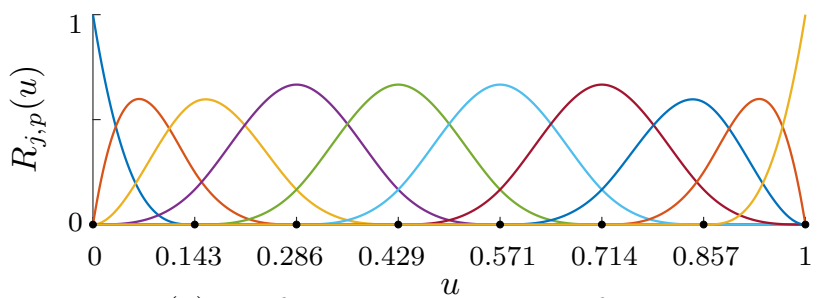

(a) Uniform parameters, uniform knots.
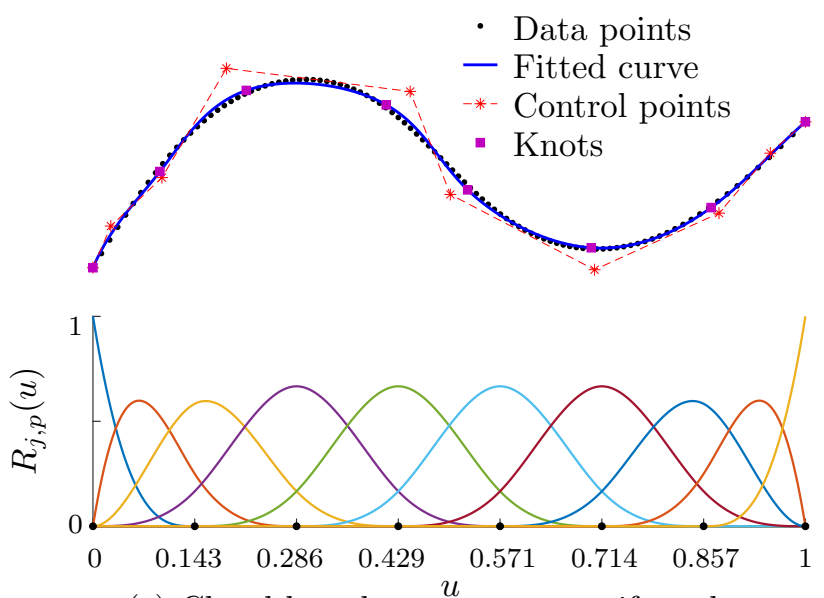

(c) Chord-length parameters, uniform knots.

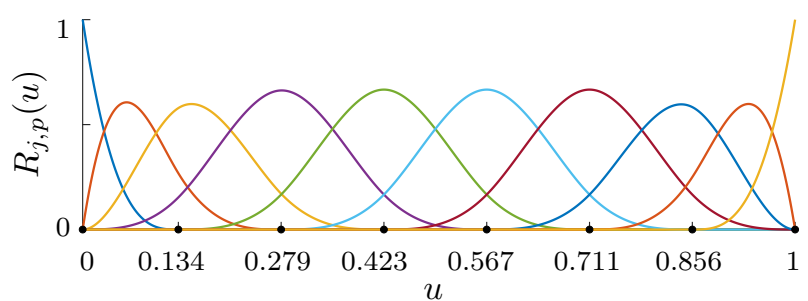

(b) Uniform parameters, De Boor's knots.
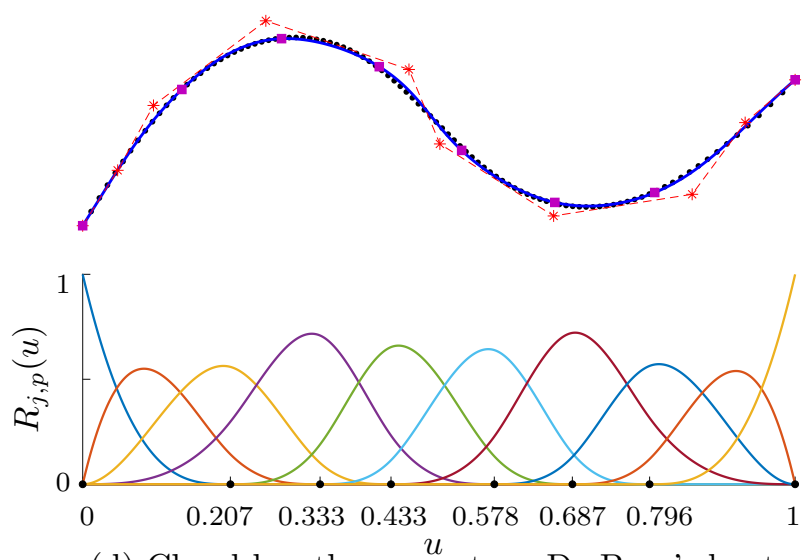

(d) Chord-length parameters, De Boor's knots.

Figure 4: Effect of different parameterizations and knot placement algorithms on curve fitting results and corresponding basis functions. 


\section{Isogeometric discretization and collocation}

By using the B-spline basis functions introduced in the previous section, the approximation of the variables of our problem discussed in Section 3 reads

$$
\begin{aligned}
\boldsymbol{\vartheta}(u) & =\sum_{j=0}^{n} R_{j, p}(u) \check{\boldsymbol{\vartheta}}_{j} \text { with } u \in I_{u}, \\
\boldsymbol{u}(u) & =\sum_{j=0}^{n} R_{j, p}(u) \check{\boldsymbol{u}}_{j} \text { with } u \in I_{u}, \\
\mathbf{m}(u) & =\sum_{j=0}^{n} R_{j, p}(u) \check{\mathbf{m}}_{j} \text { with } u \in I_{u}, \\
\mathbf{n}(u) & =\sum_{j=0}^{n} R_{j, p}(u) \check{\mathbf{n}}_{j} \text { with } u \in I_{u},
\end{aligned}
$$

where $\check{\boldsymbol{\vartheta}}_{j}$ and $\check{\boldsymbol{u}}_{j}$ are the $j$ th control variables of the kinematic fields and $\check{\mathbf{n}}_{j}$ and $\check{\mathbf{m}}_{j}$ are the internal force and moment control variables. We stress that the above fields might be discretized independently of each other, namely the discretization spaces for displacements, rotations, and stresses might not necessarily be the same [37]. However, this would imply to use different sets of collocation points with an increased computational effort. In this work we opt for the simplest solution of considering the same basis functions and collocation points for all variables. This choice is supported by the results shown in [37], where excellent convergence rates were observed also using this approach.

The derivatives with respect to the physical coordinate $s \in I_{s}=[0, L]$ need to be calculated taking into account that a change of parameterization is required since the basis functions are defined on the normalized domain $I_{u}=[0,1]$. Namely, for any vector quantity $\boldsymbol{g}: I_{u} \rightarrow \mathbb{R}^{3}$, we have $\boldsymbol{g}_{,_{s}}=\boldsymbol{g}{ }_{, u} / \jmath$, where $\jmath=d s / d u=\left\|\boldsymbol{c}_{{ }_{u}}\right\|$ is the Jacobian. Higher order derivatives, see for example Eqs. (4)-(7), are calculated using the same derivation rule. For example, the second derivative is given by $\boldsymbol{g}_{s s}=\boldsymbol{g},_{u и} / \jmath^{2}-\boldsymbol{g}_{, u}\left(\boldsymbol{c}_{, u} \cdot \boldsymbol{c}_{, u u}\right) / \jmath^{4}$, where $(\cdot)$ indicates the scalar product.

Recent studies proposed alternative choices for collocation points that, in specific situations, can achieve improved convergence rates [35, 83-85]; however, in the present study we collocate at the images of standard Greville points [1] defined as

$$
u_{i}^{c}=\frac{u_{i+1}+\ldots+u_{i+p}}{p} \text { for } i=0, \ldots, n .
$$




\subsection{Collocation of displacement-based formulation}

The $2 \times 3 \times(n-1)$ discretized and collocated equations (see Eqs. (22) and (23)) take the following form

$$
\begin{array}{r}
\sum_{j=0}^{n}\left[\mathbb{C} \widetilde{\mathbf{t}}_{1} R_{j, p}^{\prime}-\widetilde{\boldsymbol{\kappa}} \widetilde{\mathbb{t}}_{1} R_{j, p}\right]_{u=u_{i}^{c}} \check{\boldsymbol{\vartheta}}_{j}+ \\
\sum_{j=0}^{n}\left[\mathbb{C} R_{j, p}^{\prime \prime}-(\widetilde{\boldsymbol{\kappa}} \mathbb{C}+\mathbb{C} \widetilde{\boldsymbol{\kappa}}) R_{j, p}^{\prime}-\left(\mathbb{C} \widetilde{\boldsymbol{\kappa}}^{\prime}-\widetilde{\boldsymbol{\kappa}} \mathbb{C} \widetilde{\boldsymbol{\kappa}}\right) R_{j, p}^{\prime}\right]_{u=u_{i}^{c}} \check{\boldsymbol{u}}_{j}+\overline{\mathbf{n}}=\mathbf{0}, \\
\sum_{j=0}^{n}\left[\mathbb{D} R_{j, p}^{\prime \prime}-(\mathbb{D} \widetilde{\boldsymbol{\kappa}}+\widetilde{\boldsymbol{\kappa}} \mathbb{D}) R_{j, p}^{\prime}+\left(\widetilde{\boldsymbol{\kappa}} \mathbb{D} \widetilde{\boldsymbol{\kappa}}-\mathbb{D} \widetilde{\boldsymbol{\kappa}}^{\prime}+\widetilde{\mathbf{t}}_{1} \widetilde{\mathbb{C}} \widetilde{\mathbf{t}}_{1}\right) R_{j, p}\right]_{u=u_{i}^{c}} \check{\boldsymbol{\vartheta}}_{j}+ \\
\sum_{j=0}^{n}\left[\widetilde{\mathbf{t}}_{1} \mathbb{C} R_{j, p}^{\prime \prime}-\widetilde{\mathbf{t}}_{1} \mathbb{C} \widetilde{\boldsymbol{\kappa}} R_{j, p}\right]_{u=u_{i}^{c}} \check{\boldsymbol{u}}_{j}+\overline{\mathbf{m}}=\mathbf{0},
\end{array}
$$

with $i=1, \ldots n-1$. Eqs. (55) and (56) form a linear system of $2 \times 3 \times(n-1)$ equations with $2 \times 3 \times(n+1)$ unknowns. The 12 missing equations (6 per beam ends) are provided by the boundary conditions. For example, in the case of clamped end at $s=0$ (or equivalently $\left.u=u_{0}^{c}=0\right)$, the six discretized and collocated boundary equations are

$$
\begin{aligned}
& \sum_{j=0}^{n} R_{j, p}\left(u_{0}^{c}\right) \check{\boldsymbol{u}}_{j}=\mathbf{0} \\
& \sum_{j=0}^{n} R_{j, p}\left(u_{0}^{c}\right) \check{\boldsymbol{\vartheta}}_{j}=\mathbf{0} .
\end{aligned}
$$

The discretized and collocated form of the Neumann boundary conditions, e.g., considering a free end at $s=L$ (or equivalently $u=u_{n}^{c}=1$ ), reads as

$$
\begin{array}{r}
\sum_{j=0}^{n}\left[\mathbb{C}\left(R_{j, p}^{\prime}-\widetilde{\boldsymbol{\kappa}} R_{j, p}\right)\right]_{u=u_{n}^{c}} \check{\boldsymbol{u}}_{j}+\sum_{j=0}^{n}\left[\widetilde{\mathbf{t}}_{1} R_{j, p}\right]_{u=u_{n}^{c}} \check{\boldsymbol{\vartheta}}_{j}=\overline{\mathbf{n}}_{c}, \\
\sum_{j=0}^{n}\left[\mathbb{D}\left(R_{j, p}^{\prime}-\widetilde{\boldsymbol{\kappa}} R_{j, p}\right)\right]_{u=u_{n}^{c}} \check{\boldsymbol{\vartheta}}_{j}=\overline{\mathbf{m}}_{c} .
\end{array}
$$

Eqs. (55) and (56) together with Eqs. (57)-(60) form a square linear system $[6 \times(n+1)]^{2}$ which is solved for the unknowns $\check{\boldsymbol{\vartheta}}_{j}, \check{\boldsymbol{u}}_{j}$ with $j=0, \ldots, n$. 


\subsection{Collocation of mixed formulation}

The $4 \times 3 \times(n-1)$ discretized and collocated equations (see Eqs. (26)-(29)) take the following form

$$
\begin{array}{r}
\sum_{j=0}^{n}\left[R_{j, p}^{\prime}-\widetilde{\boldsymbol{\kappa}} R_{j, p}\right]_{u=u_{i}^{c}} \check{\mathbf{n}}_{j}+\overline{\mathbf{n}}=\mathbf{0}, \\
\sum_{j=0}^{n}\left[R_{j, p}^{\prime}-\widetilde{\boldsymbol{\kappa}} R_{j, p}\right]_{u=u_{i}^{c}} \check{\mathbf{m}}_{j}+\sum_{j=0}^{n}\left[\widetilde{\mathbf{t}}_{1} R_{j, p}\right]_{u=u_{i}^{c}} \check{\mathbf{n}}_{j}+\overline{\mathbf{m}}=\mathbf{0}, \\
\sum_{j=0}^{n}\left[\mathbb{C} R_{j, p}^{\prime}-\mathbb{C} \widetilde{\boldsymbol{\kappa}} R_{j, p}\right]_{u=u_{i}^{c}} \check{\boldsymbol{u}}_{j}+\sum_{j=0}^{n}\left[\widetilde{\mathbf{t}}_{1} R_{j, p}\right]_{u=u_{i}^{c}} \check{\boldsymbol{\vartheta}}_{j}-\sum_{j=0}^{n} R_{j, p}\left(u_{i}^{c} \check{\mathbf{n}}_{j}=\mathbf{0},\right. \\
\sum_{j=0}^{n}\left[\mathbb{D} R_{j, p}^{\prime}-\mathbb{D} \widetilde{\boldsymbol{\kappa}} R_{j, p}\right]_{u=u_{i}^{c}} \check{\boldsymbol{\vartheta}}_{j}-\sum_{j=0}^{n} R_{j, p}\left(u_{i}^{c}\right) \check{\mathbf{m}}_{j}=\mathbf{0},
\end{array}
$$

with $i=1, \ldots n-1$. Eqs. (61)-(64) form a linear system of $4 \times 3 \times(n-1)$ equations with $4 \times 3 \times(n+1)$ unknowns. The 24 missing equations (12 per beam ends) are provided by the boundary conditions. For example, in the case of clamped end at $s=0$ (or equivalently $u=u_{0}^{c}=0$ ), the 12 discretized and collocated boundary equations are Eqs. (57) and (58) together with the constitutive equations (63) and (64) collocated in $u_{0}^{c}$ instead of $u_{i}^{c}$. For example, assumed a free end at $s=L$ (or equivalently $u=u_{n}^{c}=1$ ), the boundary conditions are given by Eqs. (59) and (60) complemented with Eqs. (63) and (64) collocated in $u_{n}^{c}$ instead of $u_{i}^{c}$. These boundary conditions, together with Eqs. (61)-(64), form a square linear system with dimension $[12 \times(n+1)]^{2}$ which is solved for the unknowns $\check{\boldsymbol{\vartheta}}_{j}, \check{\boldsymbol{u}}_{j}, \check{\mathbf{m}}_{j}, \check{\mathbf{n}}_{j}$ with $j=0, \ldots, n$.

Note that the primal and mixed formulations discussed above are different from those proposed in $[48,50]$ not only because their validity is restricted to geometrically linear problems, but also because they are formulated in the local (Frenet) frame. Here, the rotation operator (an element of $\mathrm{SO}(3)$ used to describe the rotation of the beam cross section) is only used in the post-process phase to transform the vector components of the solution from the local to the global frame.

\section{Numerical experiments}

Before proceeding with results, we first provide some general information that are common to all test cases. 
In all examples, a circular cross-section of $0.1 \mathrm{~m}$ radius is assumed. In addition, the Young modulus and the Poisson ratio of all curved beams are assumed to be $E=200 \mathrm{GPa}$ and $\nu=0.3$, respectively, while the shear modulus is calculated as $G=E / 2(1+\nu)$. To reconstruct the geometry of all case studies, a set of 1000 input data points are considered, obtained by the respective analytical equations. Since an analytical solution does not exist for the considered curved beam examples, the IGA-C computations are compared with "overkill" finite element results, obtained with the commercial software ABAQUS by generating appropriate meshes of quadratic beam elements and requiring a convergence up to six decimal places. The tip loads and reference tip displacements are reported in Table 1. Note that for all the analyzed problems, the displacements are small enough to allow geometrically linear formulations to be adopted. Finally, in all examples, we used a code in the form of "APK" to indicate different combinations of parameterization and knot placement techniques discussed in Section 4. In this coding system, P refers to the parameterization and takes values 1 or 2 for chord-length or equally spaced methods, respectively; whereas $\mathrm{K}$ refers to the knot placement and takes values 1 or 2 for the uniformly spaced or De Boor's methods, respectively. Note that when the equally spaced parameterization is used, there is no difference between uniform and De Boor knot placement techniques. Therefore, in total we will analyze three different cases: A11, A12, and A2 (=A21=A22).

Table 1: The tip loads and reference tip displacements of the studied examples computed by overkill FEA in ABAQUS environment.

\begin{tabular}{lll}
\hline & Tip Force $(\mathrm{N})$ & Tip displacement $(\mathrm{mm})$ \\
\hline Tschirnhausen beam & $-[0,200,0]^{\top}$ & {$[0.902449,-4.083810,0]^{\top}$} \\
Lissajous beam & {$[0,0,200]^{\top}$} & {$[0.131965,-0.104978,0.433117]^{\top}$} \\
Viviani beam & {$[0,0,200]^{\top}$} & {$[0.227786,-0.117027,0.238018]^{\top}$} \\
Logarithmic spiral beam & {$[0,200,0]^{\top}$} & {$[1.879695,9.436861,-0.188030]^{\top}$} \\
\hline
\end{tabular}

\subsection{The Tschirnhausen planar beam}

The Tschirnhausen beam is a well-known planar structure with variable curvature that is studied frequently in the literature (see, e.g., $[86,87]$ ). The geometry of the beam is 
defined analytically by Eq. (65). The beam model is depicted in Figure 5 assuming that it is clamped at the right end and is subject to an in-plane tip load of $[0,-200,0]^{\top} \mathrm{N}$ at the left end.

$$
\left\{\begin{array}{l}
x=3\left(3-\zeta^{2}\right) \\
y=\zeta\left(3-\zeta^{2}\right)
\end{array} \quad 0 \leq \zeta \leq \sqrt{3}\right.
$$

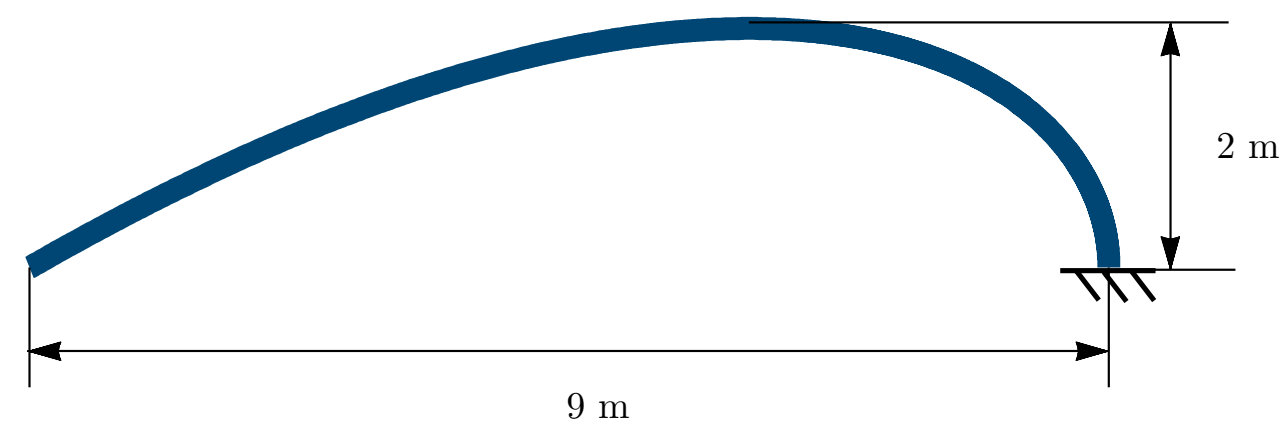

Figure 5: Tschirnhausen free-form curved beam.

Figure 6 illustrates the convergence curves of the relative error versus the number of collocation points for the Tschirnhausen beam obtained by both displacement-based and mixed formulations.

We observe that case A2, corresponding to the equally spaced parameterization method with either uniform or De Boor knot placements, outperforms the other combinations. High accuracy is already obtained even with the primal formulation with a pretty coarse mesh $(n=20)$. Both degree elevation in the displacement-based formulation and the use of a mixed formulation significantly improve the convergence quality.

The poorer performances of cases A11 and A12, namely chord-length parameterization combined with either uniform or De Boor knot placements, especially for $p=4$, is related to the parameterization. Although A11 and A12 are expected to have a constant Jacobian, a deeper examination reveals that chord-length parameterization introduces small instabilities in the Jacobian which affect the quality of the convergence of the error. More details are given in Appendix A, in particular see Figure A.13. Another reason for the poorer convergence behavior of A11 and A12 with respect to A2 is the much higher error in the geometry 


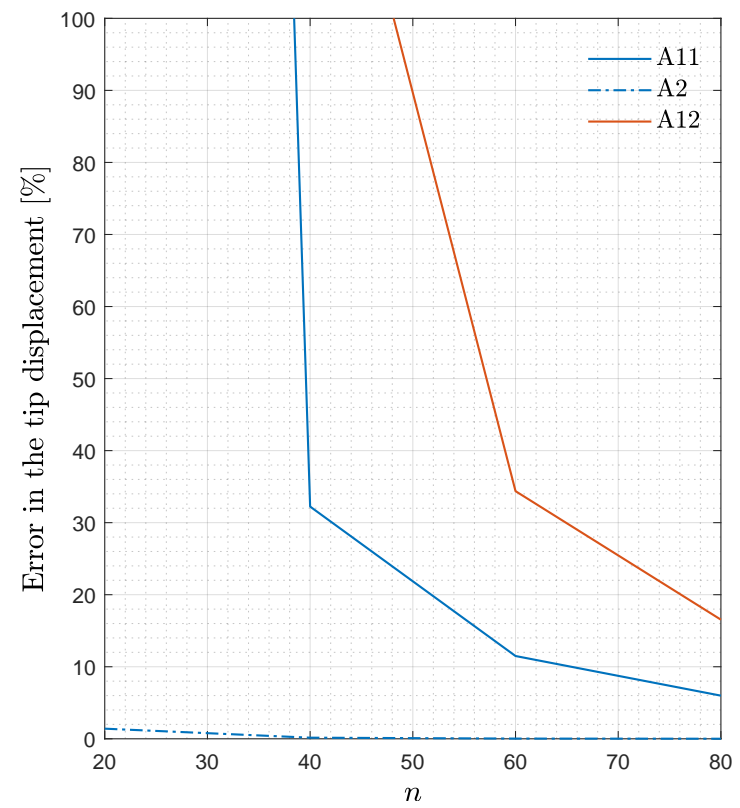

(a) Disp.-based.

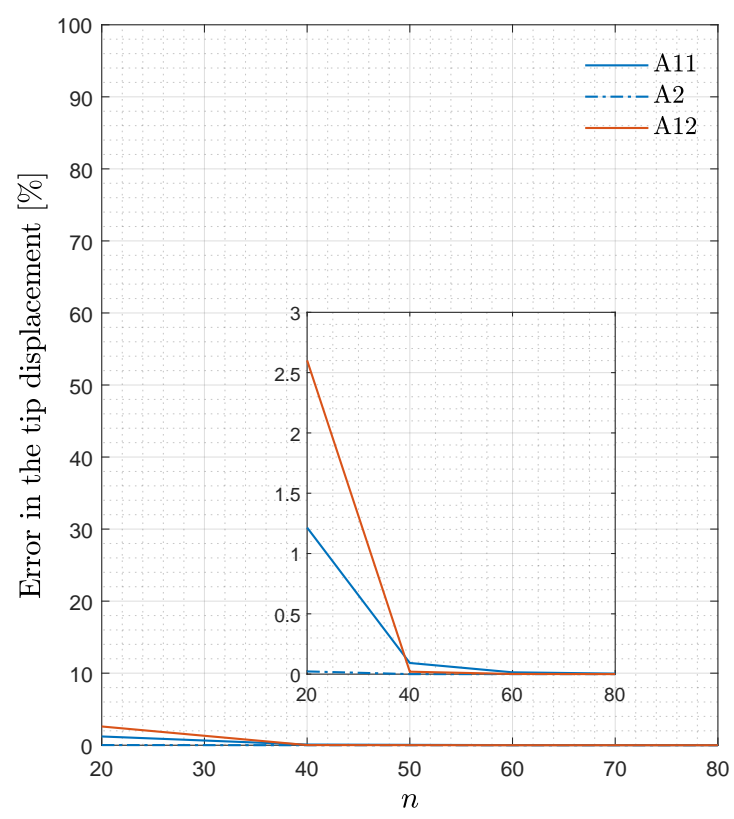

(c) Disp.-based.

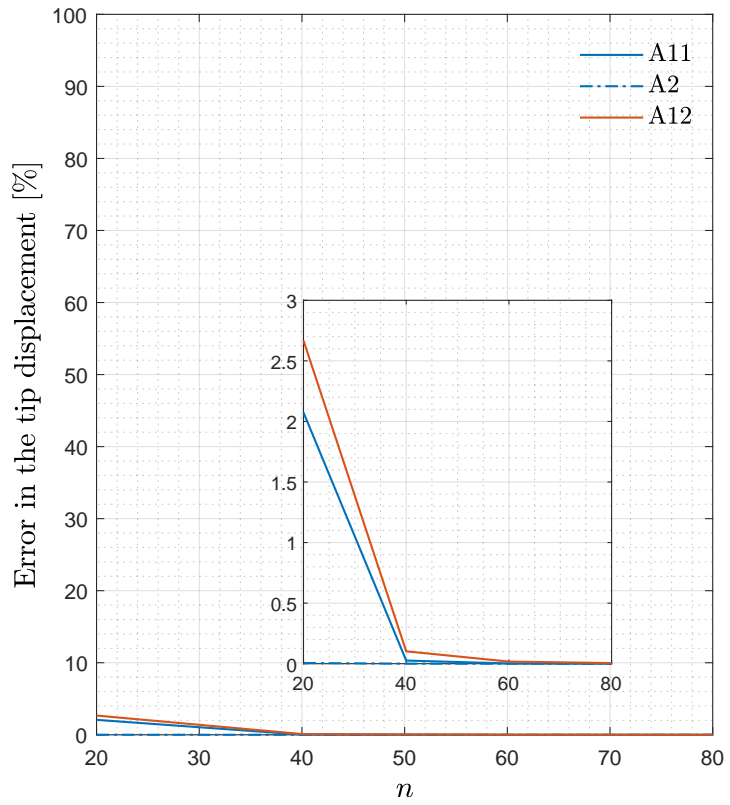

(b) Mixed.

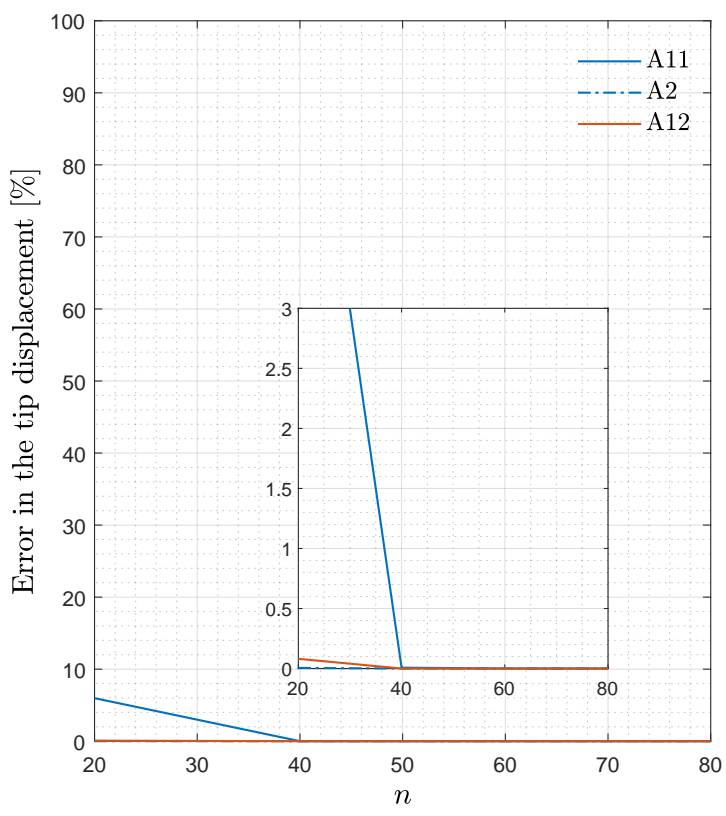

(d) Mixed.

Figure 6: Error in \% versus number of collocation points for the Tschirnhausen beam: displacement-based ((a) and (c)), and mixed ((b) and (d)) formulations with B-spline basis functions with degree $p=4$ upper and $p=6$ lower plots.

approximation. While A2 guarantees a least-square error (see Eq. (46)) smaller than 10-10, A11 and A12 approximate the geometry with an error several orders of magnitude larger 
(see Figure B.20 in Appendix B).

\subsection{The Lissajous spatial curved beam}

The Lissajous curved beam is a complex harmonic function in space that is described by the following analytical equations

$$
\left\{\begin{array}{l}
x=\cos 3 \zeta \\
y=\sin 2 \zeta \\
z=\sin 7 \zeta
\end{array} \quad-\pi / 3 \leq \zeta \leq \pi / 3 .\right.
$$

The beam is clamped at one end (see Figure 7) and is subject to a tip load $[0,0,200]^{\top} \mathrm{N}$ in the $z$-direction at the free end. Figure 8 shows the convergence curves of the relative error versus the number of collocation points. The complexity of the Lissajous geometry requires a high approximation degree to properly represent the fourth-order derivative terms appearing in the displacement-based formulation (see Eq. (7)). Figure 8a indeed reveals that degree $p=4$ is not suitable for this geometry. With the primal formulation, $p=6$ offers a significant improvement in the case A2 (see Figure 8c) still with a residual error of $\sim 2 \%$ for the finest mesh. In the mixed formulation, where only third-order derivatives are needed (see Eq. (5)), $p=4$ becomes appropriate for the problem. Chord-length parameterization, especially when combined with uniform knots (see case A11), exhibits the worst performance even with $p=6$. As in the previous test case, such a poor and nonuniform convergence quality is caused by the instabilities appearing in the Jacobian (even more evident in this test case, see Figures A.14 and A.15 in Appendix A for more details) together with orders of magnitude higher error in the geometry reconstruction (see Figure B.21 in Appendix B). For $p=6$ and $n=120$ the instabilities are more severe than $p=4$ and this might explain why in the mixed formulation the case with $p=6$ behaves poorer than the case with $p=4$ (compare Figures 8b and 8d). Instead, the better performance of A2 with $p=4$ versus A2 with $p=6$ is not fully understood at this stage and would require further investigations.

Moreover, it is noted that, as opposed to A12 and A2, which result to have a larger number of collocation points over the regions of the physical domain $I_{s}$ where strong and localized variations of curvature and torsion occur, combination A11 is characterized by a 
uniform distribution of collocation points, which is particularly unfavorable when complex geometries are concerned.

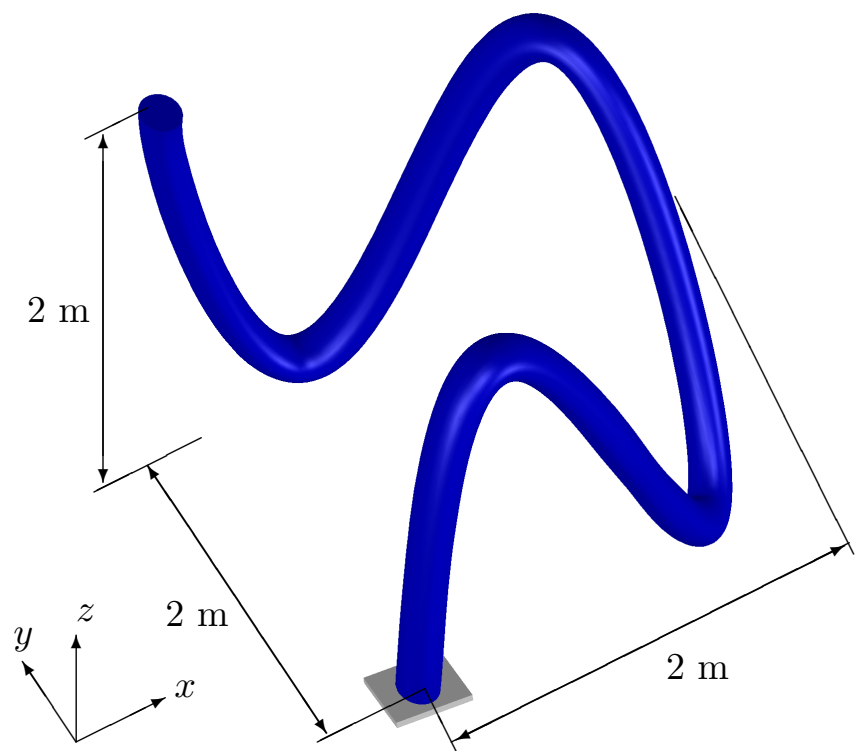

Figure 7: The Lissajous free-form curved beam.

\subsection{The Viviani curved beam}

The structural behavior of the Viviani curved beam [88] under a tip load is investigated in this section. The geometry of this spatial curved beam is built from the intersection curve of a sphere of radius $2 a$ centered at the origin with a cylinder of radius $a$ centered at $(a, 0,0)$. The analytical formulation of the geometry and the clamped-free configuration of the beam is represented by Eq. (67) and Figure 9, respectively, considering $a=1 \mathrm{~m}$. The beam is subject to a tip load $[0,0,200]^{\top} \mathrm{N}$ in the $z$-direction at the free end.

$$
\left\{\begin{array}{l}
x=a(1+\cos \zeta) \\
y=a \sin \zeta \\
z=2 a \sin (\zeta / 2)
\end{array} \quad-\pi \leq \zeta \leq \pi .\right.
$$

Figure 10 shows the convergence curves for the Viviani beam. In the primal formulation, $p=4$ is again unsuitable to properly describe the variations of curvature and torsion. With $p=6$, a significant improvement is obtained (see Figure 10c) for all combinations of parameterization and knot insertion techniques. Once more A2 yields the the best result. 


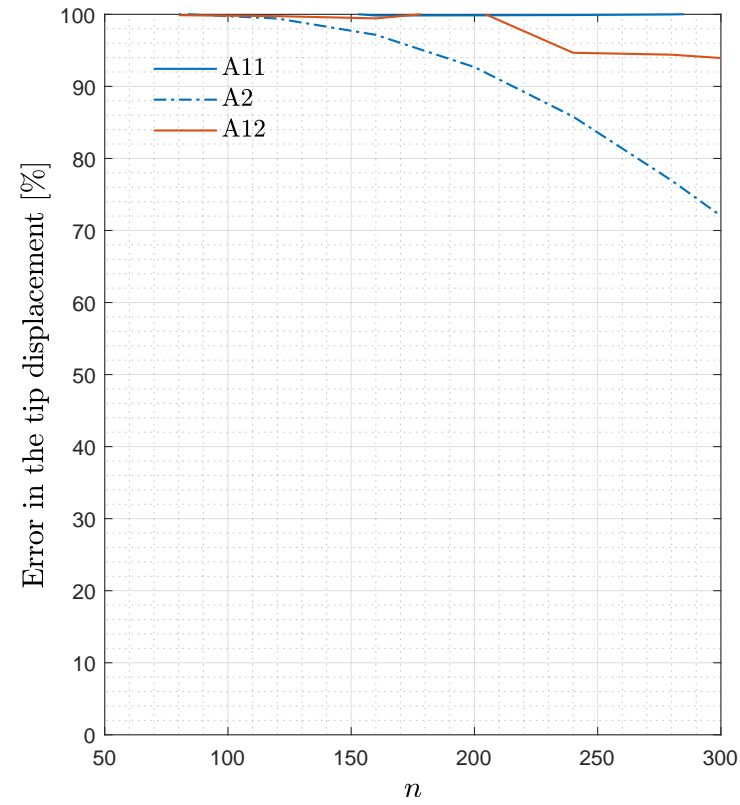

(a) Disp.-based.

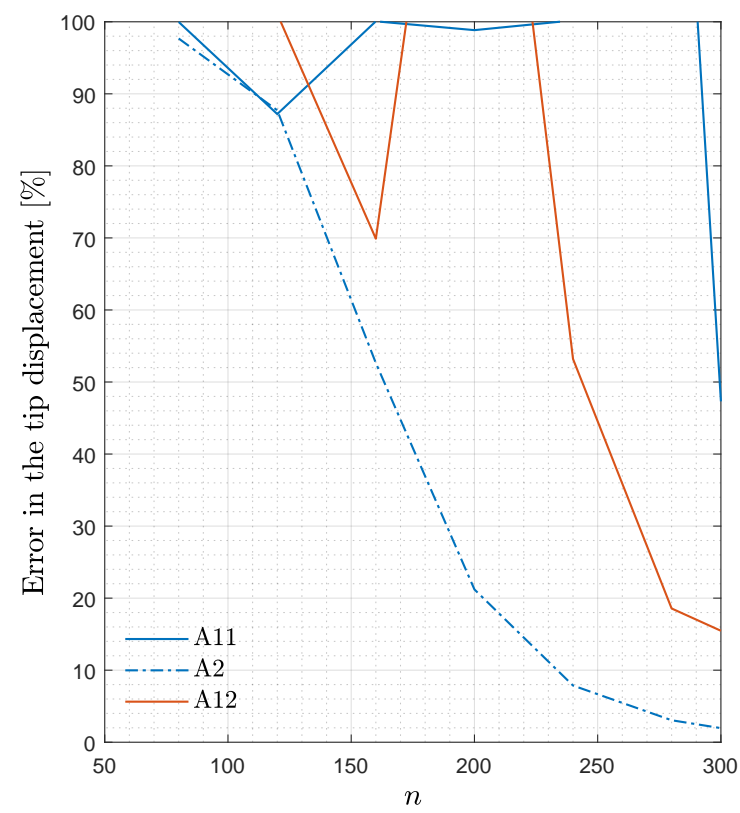

(c) Disp.-based.

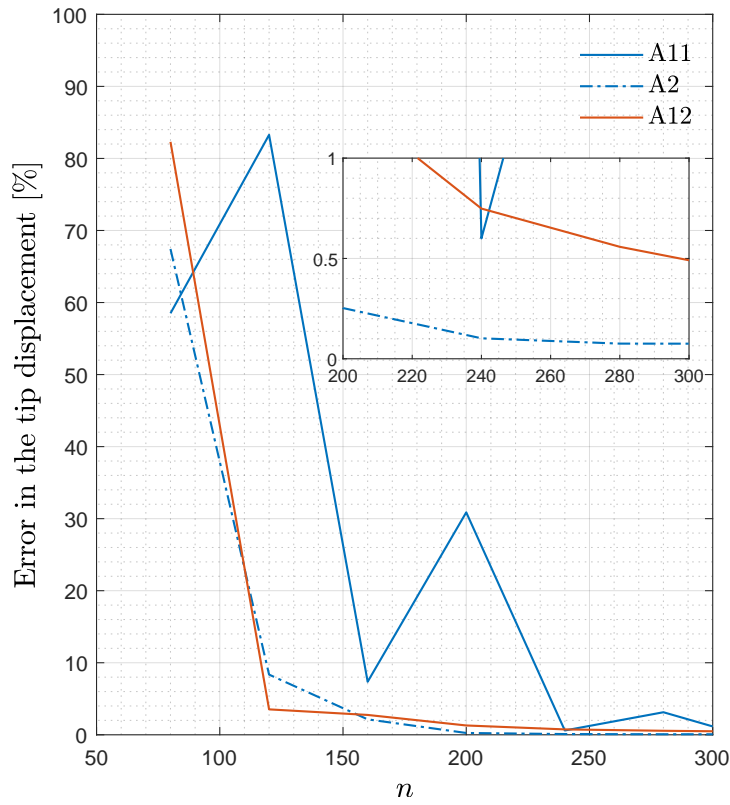

(b) Mixed.

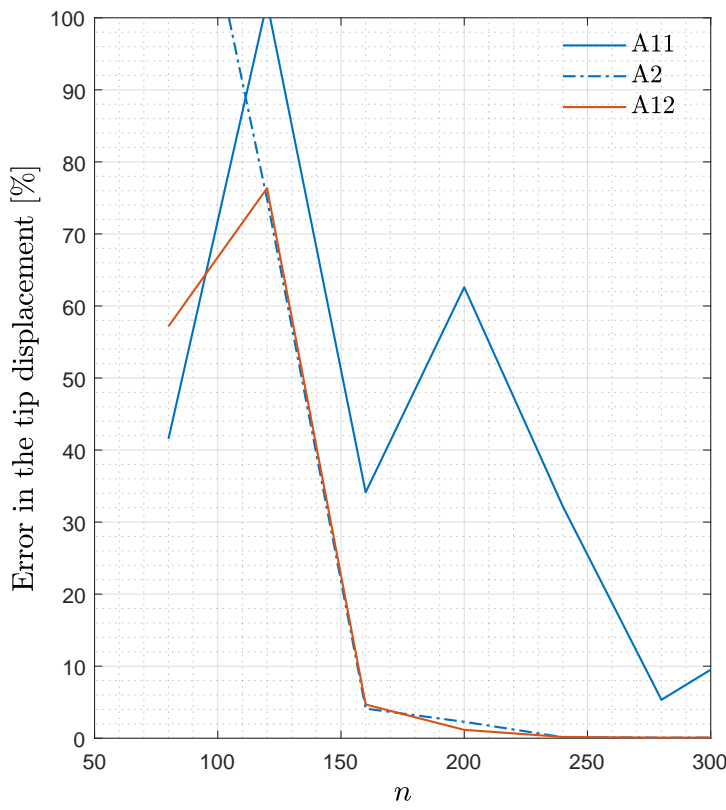

(d) Mixed.

Figure 8: Error in \% versus number of collocation points for the Lissajous beam: displacement-based ((a) and (c)), and mixed ((b) and (d)) formulations with basis functions of degree $p=4$ upper and $p=6$ lower plots.

The same trend is observed also in the mixed formulation for both degrees. With $p=6, \mathrm{~A} 2$ reaches and error of $\sim 0.2 \%$ with only 30 collocation points, wheres with $p=4$ the same error 


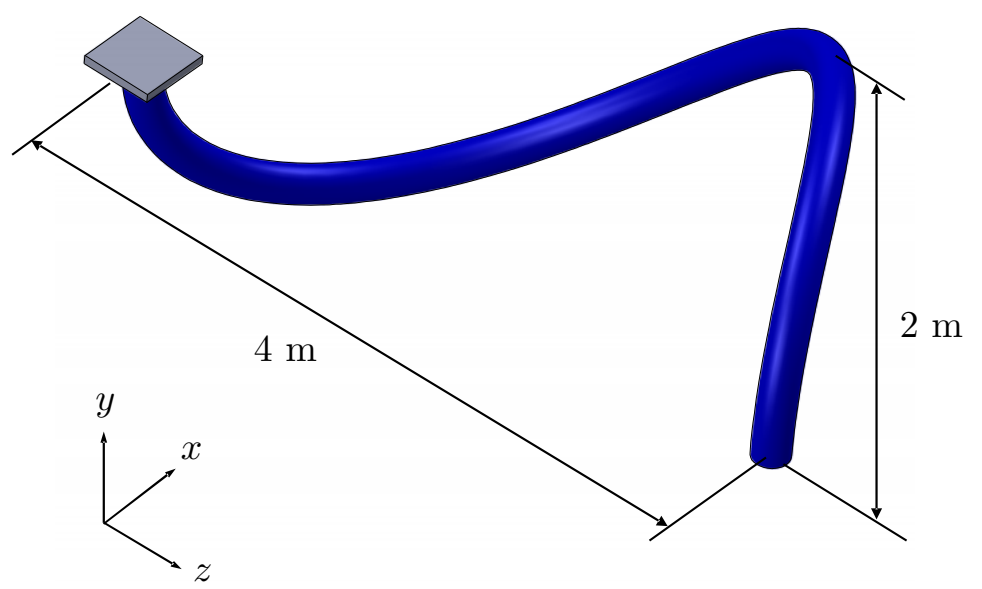

Figure 9: The clamped-free Viviani curved beam in 3D space.

is reached with 60 collocation points. Finally we observe that A11 and A12 do not perform as bad as in the Lissajous case. This is due to three main reasons: a slower and weaker variation of curvature and torsion; the presence of no (for $p=6$ ) or negligible (for $p=4$ ) instabilities in the Jacobian (see Figures A.16 and A.17 in Appendix A); a much similar behavior of the error in the geometry approximation for all three cases (see Figure B.22 in Appendix B).

\subsection{The logarithmic spiral curved beam}

In the final test case of this paper we investigate the IGA-C results of an out-of-plane logarithmic spiral beam subjected to a tip load of $[0,200,0]^{\top} \mathrm{N}$. The centroid line of this cantilever beam (see Figure 11) is a curve with the following analytical expression:

$$
\left\{\begin{array}{l}
x=2 \cos \zeta e^{\zeta / 2} \\
y=2 \sin \zeta e^{\zeta / 2} \quad-2.35 \pi \leq \zeta \leq 0.85 \pi . \\
z=\zeta / 10
\end{array}\right.
$$

Figure 12 shows the convergence curves of the relative error versus the number of collocation points. It is remarked that in this case curvature and torsion vary very strongly and rapidly nearby the clamped end and rather slowly nearby the free end, where they tend to zero.

In the primal formulation A2 blows up. This happens because the system becomes 


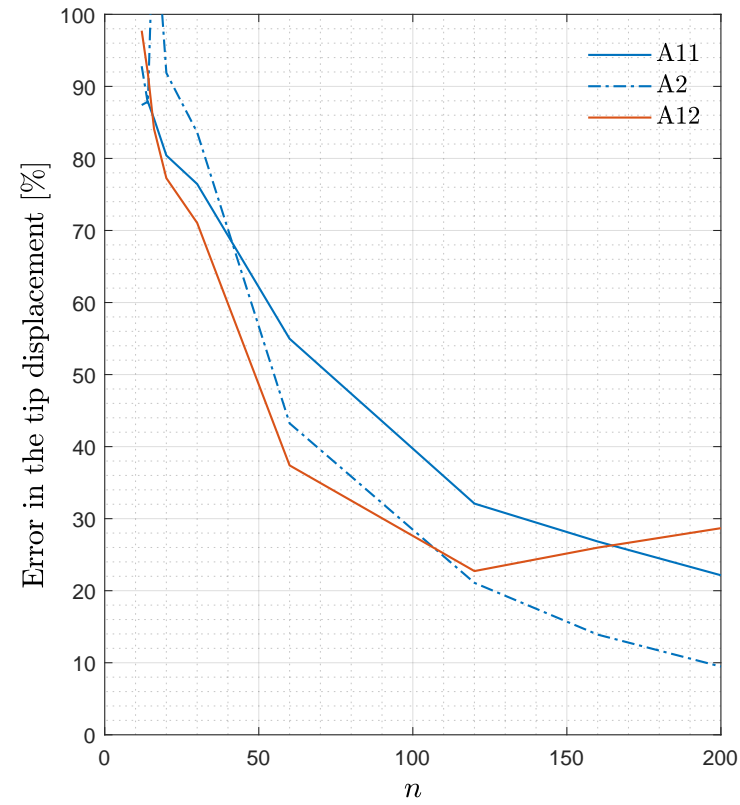

(a) Disp.-based.

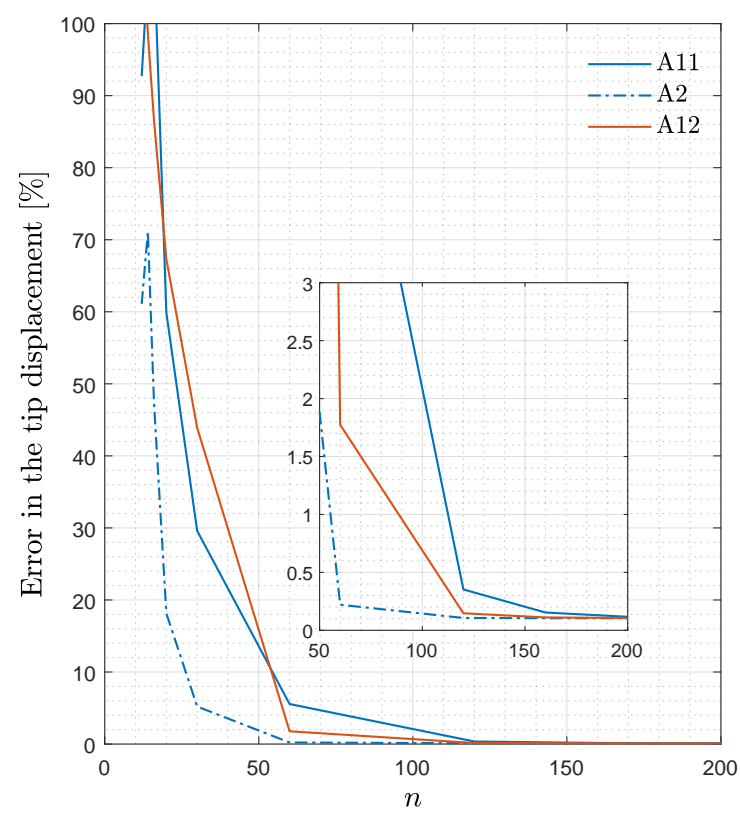

(c) Disp.-based.

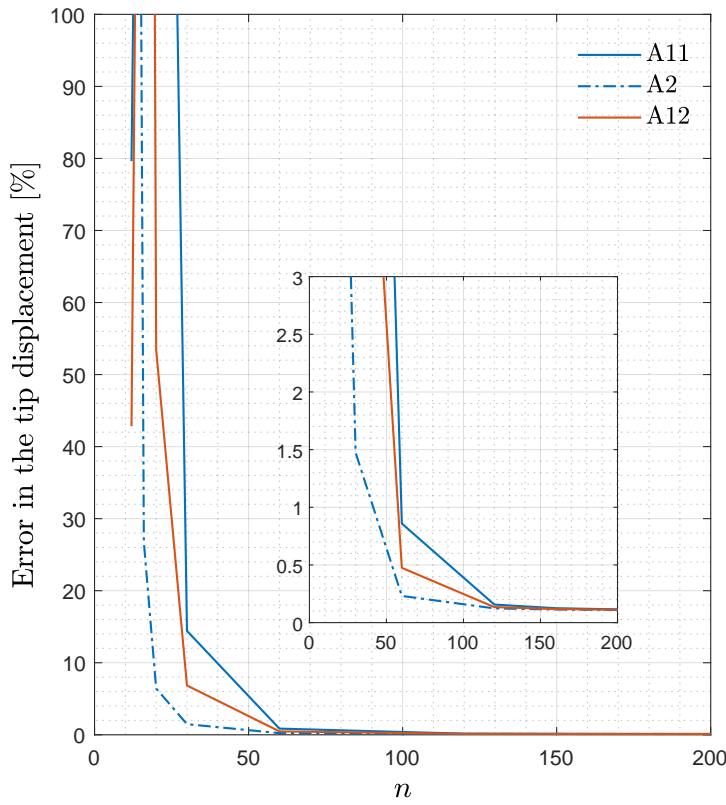

(b) Mixed.

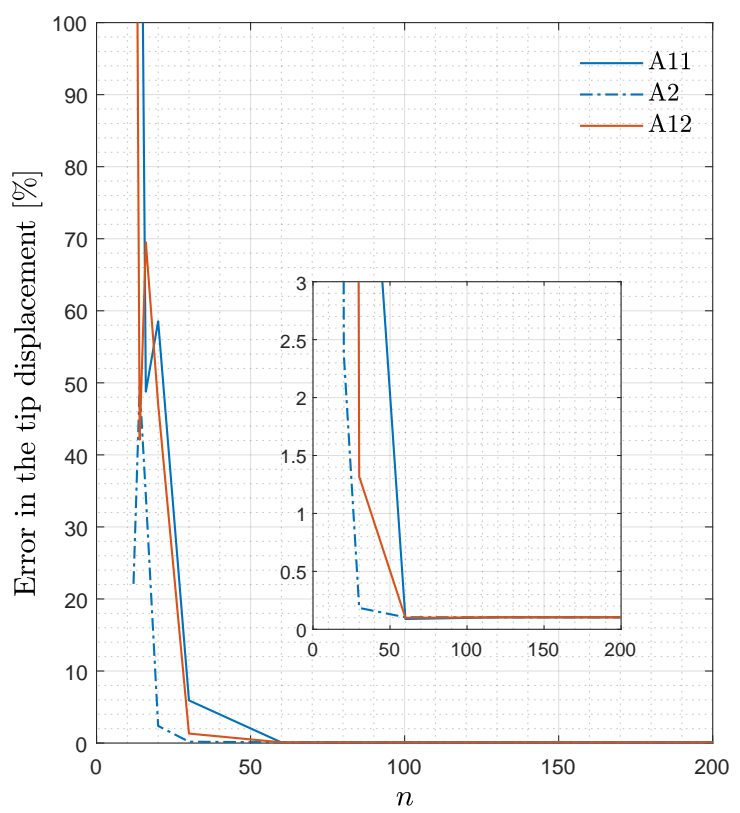

(d) Mixed.

Figure 10: Error in \% versus number of collocation points for the Viviani beam: displacement-based ((a) and (c)), and mixed ((b) and (d)) formulations with basis functions of degree $p=4$ upper and $p=6$ lower plots. 


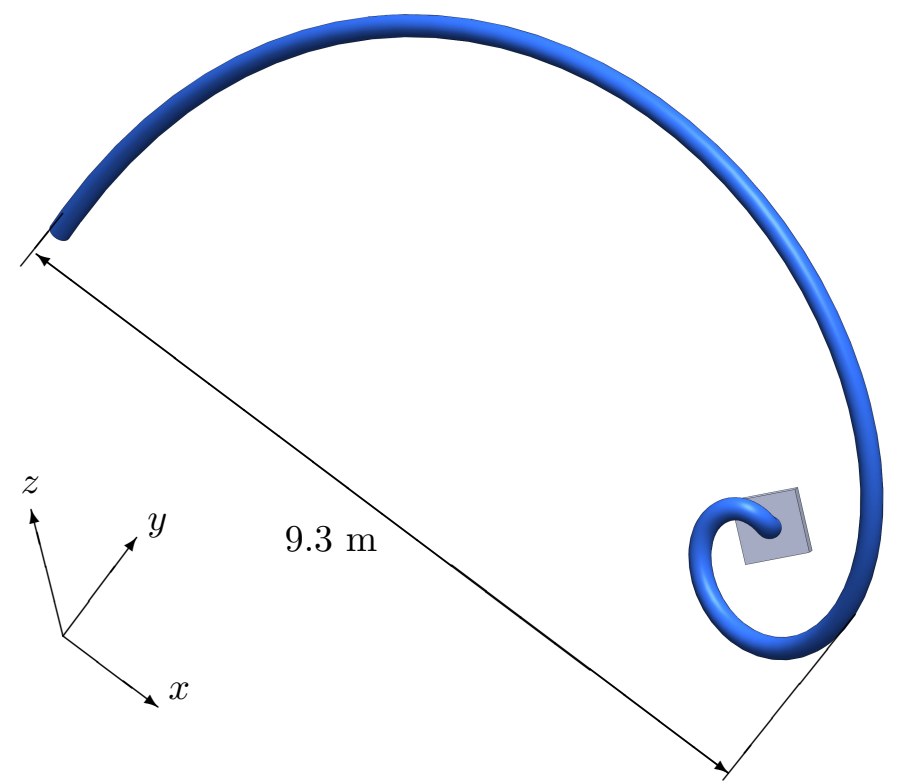

Figure 11: The out-of-plane logarithmic spiral free-form curve beam.

ill-conditioned. This is possibly caused by the fact that with A2 the Jacobian grows very rapidly in the same regions where curvature and torsion become very small, see Figure A.18a. We recall that in the displacement-based formulation the Jacobian raised to the power of eight appears in the calculation of fourth-order derivatives. The poor performance of A11 and A12, similarly to the previous cases, are caused by the instabilities appearing in the Jacobians (see Figure A.18 in Appendix A) and, as the number of control points increases, by the instability in the geometry fitting error: the system to reconstruct the geometry (see Eq. (47)) becomes ill-conditioned in case A11 (see Figure B.23 in Appendix B). A higher degree ( $p=6$, see Figure 12c) produces a significant improvement on A2 that does not crash anymore and performs very well.

In the mixed formulation, for $p=4, \mathrm{~A} 2$ is the best-preforming parameterization reaching an error level of $\sim 0.4 \%$ with $n=60$. Also A12 exhibits a good convergence curve, while A11 is again the worst case. The same trend is observed with $p=6$ (see Figure 12d). A2 reaches an error of $0.007 \%$ already with 60 collocation points, while A12 requires 140 points to reach the same error. A11 crashes for the same reasons of the primal formulation. 


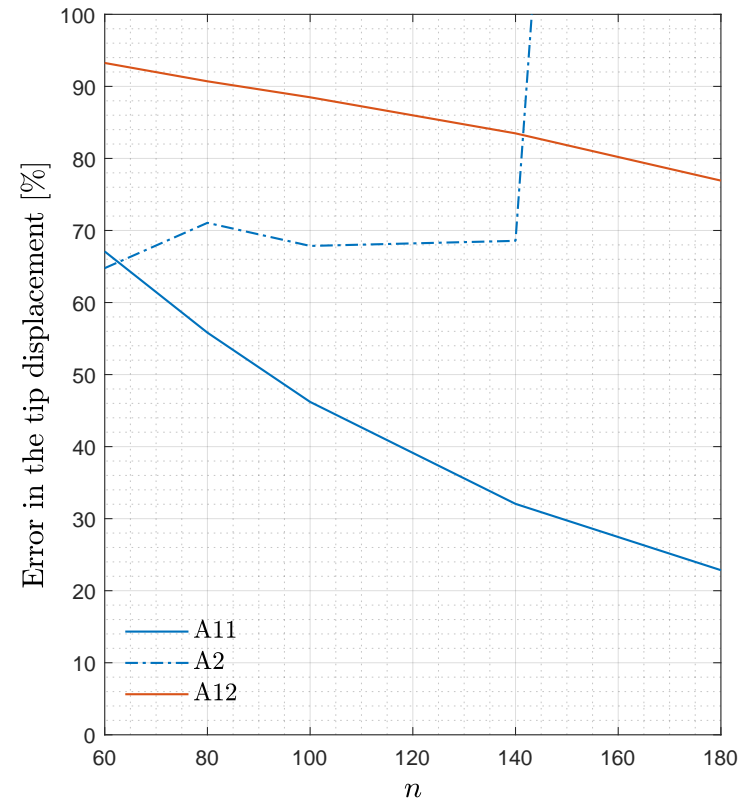

(a) Disp.-based.

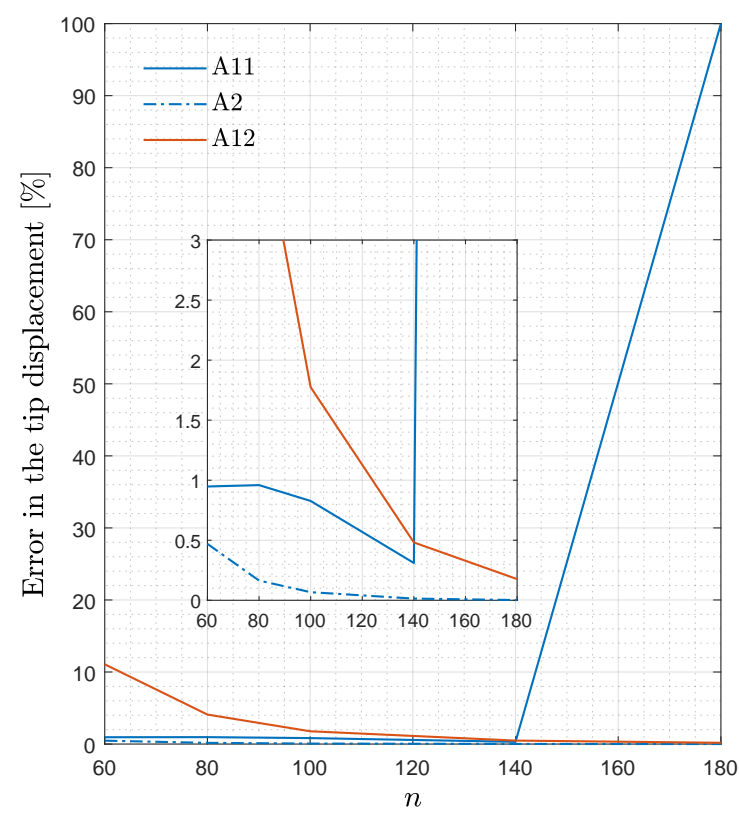

(c) Disp.-based.

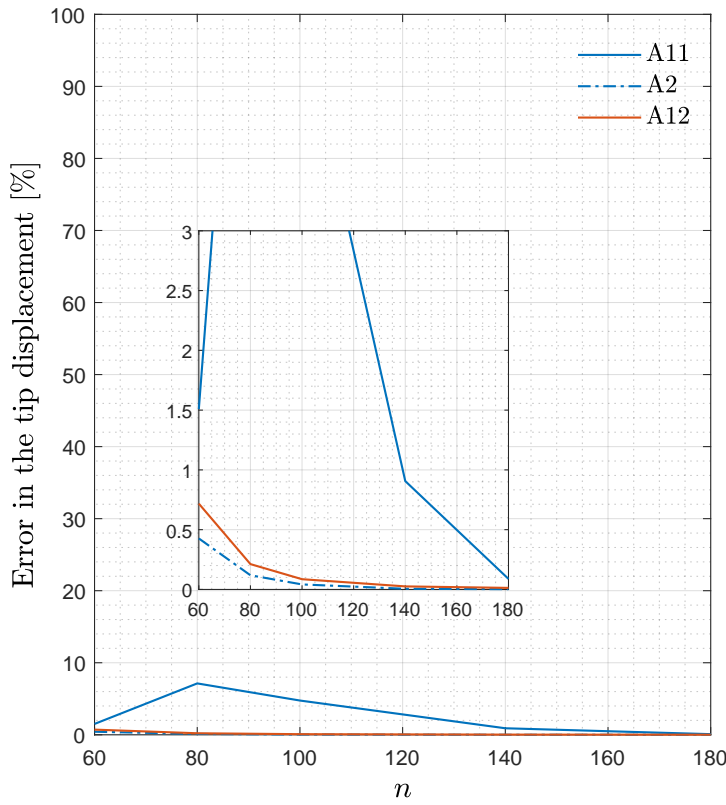

(b) Mixed.

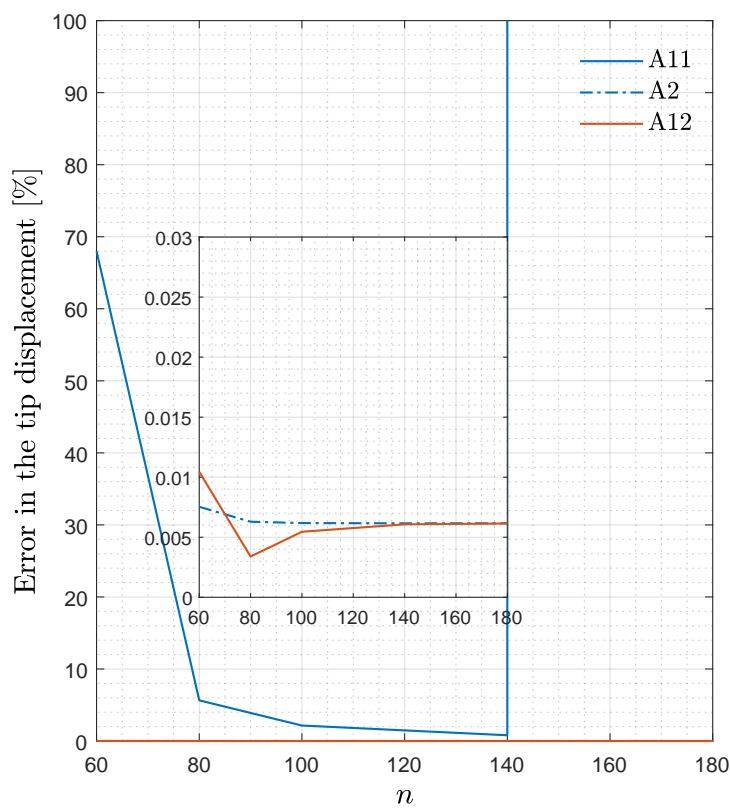

(d) Mixed.

Figure 12: Error in \% versus number of collocation points for the spiral beam: displacement-based ((a) and $(\mathrm{c})$ ), and mixed ((b) and (d)) formulations with basis functions of degree $p=4$ upper and $p=6$ lower plots. 


\section{Conclusions}

We have presented a displacement-based and a mixed IGA-C formulation for threedimensional, shear-deformable beams with highly curved geometries. The strong form of the governing equations has been derived in a compact form through the definition of two matrix operators conveniently used to perform first and second order derivatives of the vector fields involved in the formulations. Both primal and mixed formulations are derived in the space-varying Frenet local frame. Transformation of the results into the fixed global Cartesian frame is made at the end as a post-process. This approach turned out to be very efficient and easy to implement within a collocation-based scheme.

The simulation of highly curved three-dimensional beams raises the issue of "analysisaware modeling", namely the construction of IGA-optimal data which have a direct effect on the accuracy (e.g., knots distribution). Although IGA-C has been so far successfully applied to a wide range of problems, no existing study has been devoted to understanding the effects that different parameterization and knot placement techniques may have on the accuracy of collocation-based formulations. To fill this gap, in this work the primal and mixed IGA-C formulations have been used combining two parameterization methods (referred to as chordlength and equally spaced, respectively) with two knot placement techniques (referred to as uniformly spaced and De Boor, respectively). Through the application of the IGA-C formulations to four test cases with challenging geometries, the following main observations have been made:

- The chord-length parameterization exhibits the poorest behavior. Especially when combined with the uniformly spaced knot placement technique, it yields nonuniform convergence (or even no convergence) of the error. This is due to multiple factors, such as the numerical instabilities appearing in the Jacobian, the generally large error in the geometry approximation, the uniform distribution of collocation points (only when combined with uniform knots). With a basis functions degree appropriate to the considered geometry, chord-length parameterization delivers superior results when combined with De Boor knot placement technique.

- The equally spaced parameterization is, in most of the cases, the optimal choice. The 
geometry approximation error is always smaller compared to other combinations and no instabilities occur in the Jacobians. Only one exception has been found, namely when the Jacobian becomes extremely high and curvature and torsion tend to zero (spiral beam case). In these circumstances, equally spaced parameterization may become unstable. Nevertheless, we observed that degree elevation effectively fixed this deficiency. Since in collocation degree elevation comes almost at no additional computational cost, this is a rather interesting attribute.

The overall conclusion of this work, although further investigations will be needed, is that with the primal formulation an equally spaced parameterization is definitively the most recommended choice and, due to the high-order derivatives involved in the governing equations, it must always be used with an approximation degree of, at least, $p=6$. Some caution must be adopted when very high Jacobians and small curvatures occur. The same holds for the mixed formulation, with the difference that $p=4$ is enough to yield accurate results since only third-order derivatives are involved in the formulation. This conclusion is in sharp contrast to the results obtained with Galerkin-based formulations. This is due to the much higher sensitivity of the collocation method to the local instability detected in the Jacobian and to the direct effect the different parameterizations and knot placements have on the distribution of the collocation points.

\section{Acknowledgments}

Ali Hashemian was partially funded by the BCAM "Severo Ochoa" accreditation of excellence, Spain (SEV-2017-0718), and the Basque Government, Spain through the BERC 2018-2021 program.

Alessandro Reali was partially supported by the MIUR-PRIN project XFAST-SIMS, Italy (no. 20173C478N).

\section{Appendix A. Jacobian, curvature and torsion}

In this appendix, we report, for each test case studied in Section 6, some figures showing the variation of the Jacobian over the parametric domain $I_{u}$. The numerical oscillations 
occurring in the case of chord-length parameterization can be clearly observed. On the same figures, we add the variation of curvature, torsion, and their derivatives (to be read on the right-hand vertical axis).

\section{Tschirnhausen beam case}

Although they are extremely small, in the neighborhood of $u=0$ some instabilities are observed for cases A11 and A12, whereas a smooth Jacobian is observed in case A2. See Figure A.13.

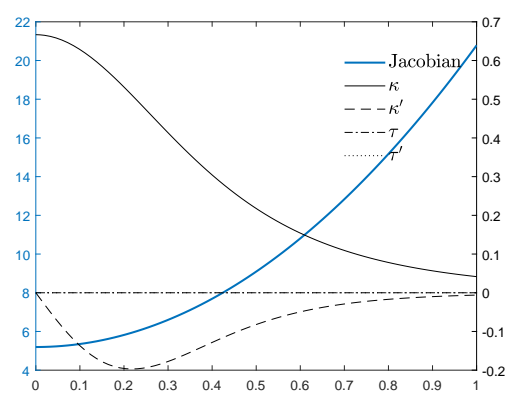

(a) Case A2.

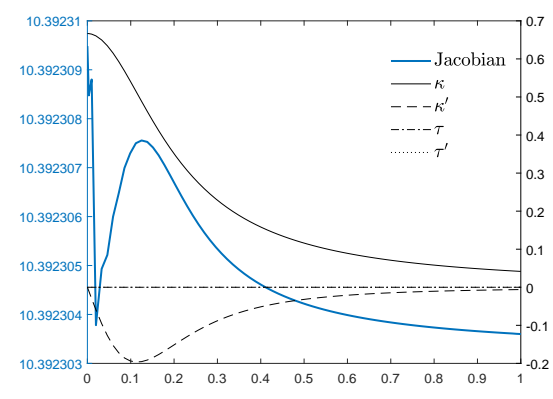

(b) Case A11.

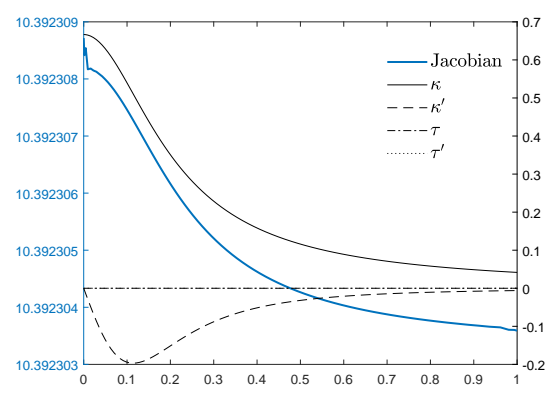

(c) Case A12.

Figure A.13: Tschirnhausen beam. $p=4, n=80$.

\section{Lissajous beam case}

For cases A11 and A12, the oscillations in the Jacobian are concentrated in correspondence of the maximum values of the curvature. Moreover, it is noted that for $p=6$ and $n=120$ the instabilities are more severe than for $p=4$.

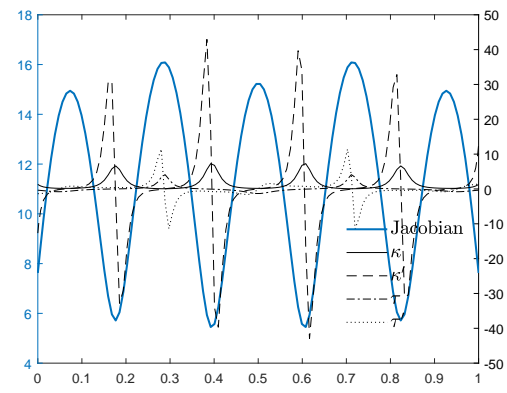

(a) Case A2.

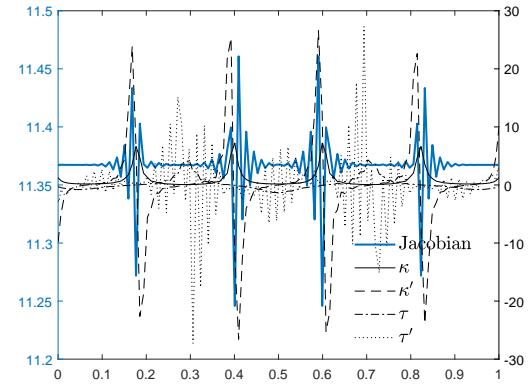

(b) Case A11.

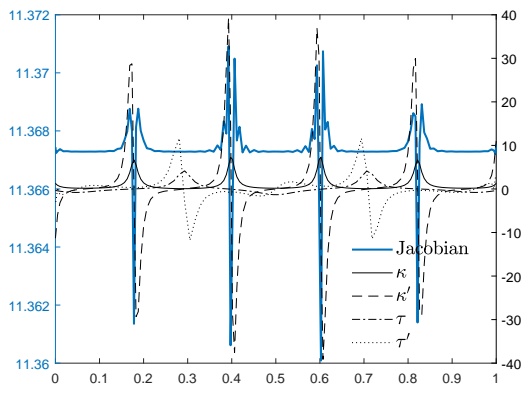

(c) Case A12.

Figure A.14: Lissajous beam. $p=4, n=120$. 


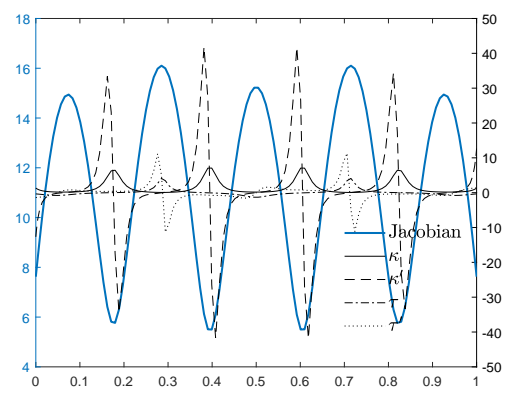

(a) Case A2.

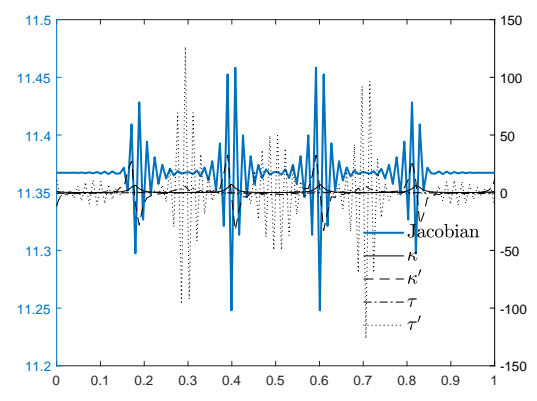

(b) Case A11.

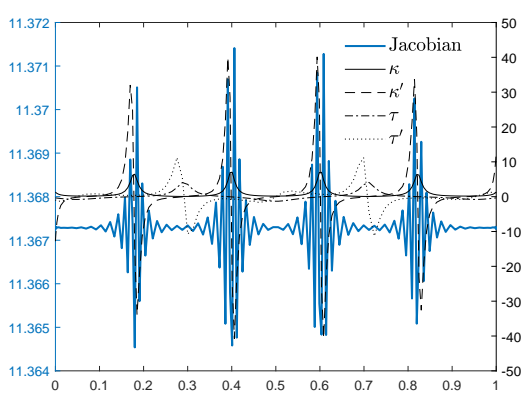

(c) Case A12.

Figure A.15: Lissajous beam. $p=6, n=120$.

413

414

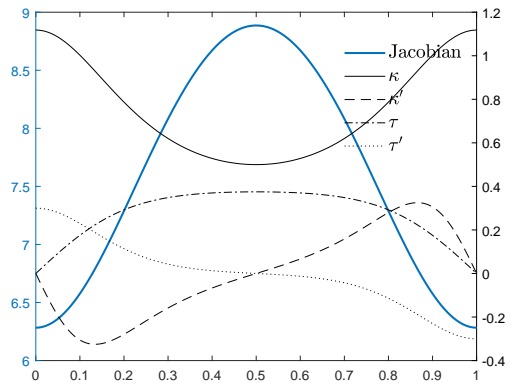

(a) Case A2.

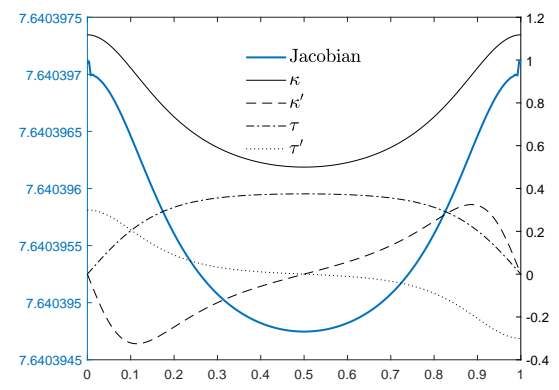

(b) Case A11.

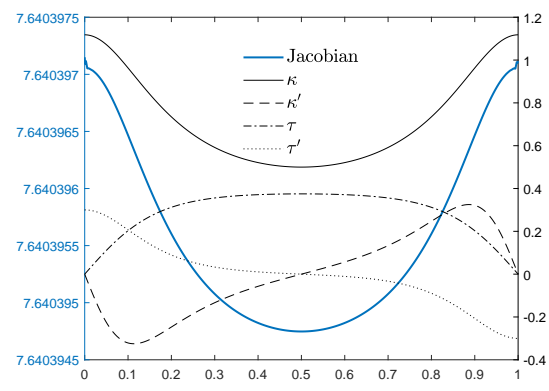

(c) Case A12.

Figure A.16: Viviani beam. $p=4, n=200$.

415

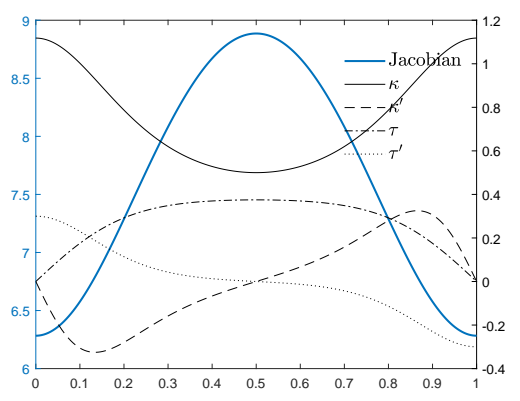

(a) Case A2.

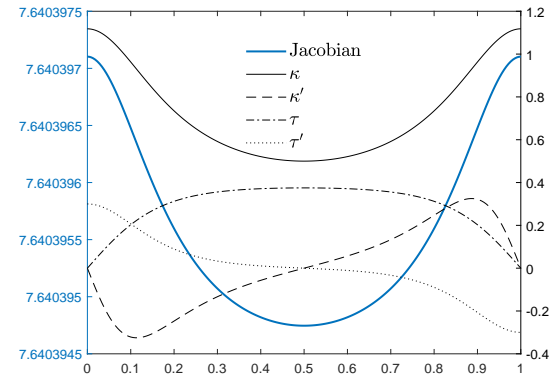

(b) Case A11.

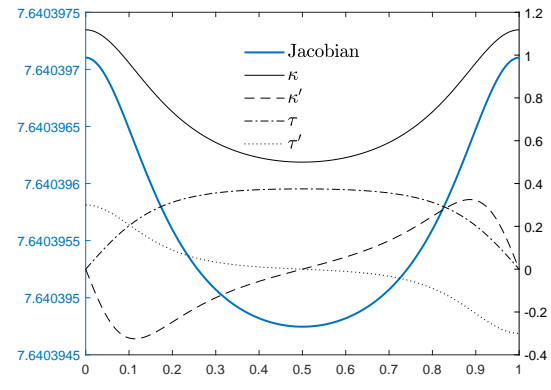

(c) Case A12.

Figure A.17: Viviani beam. $p=6, n=200$. 


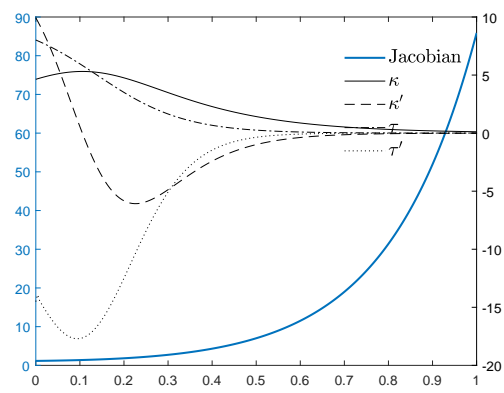

(a) Case A2

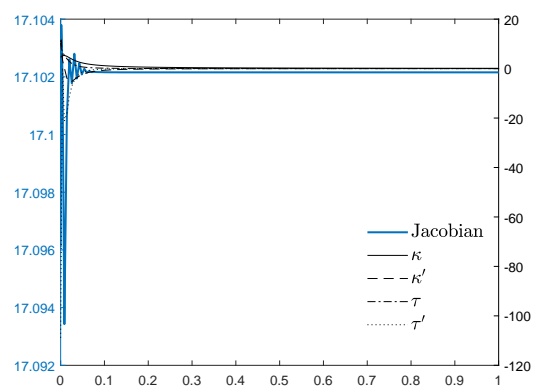

(b) Case A11.

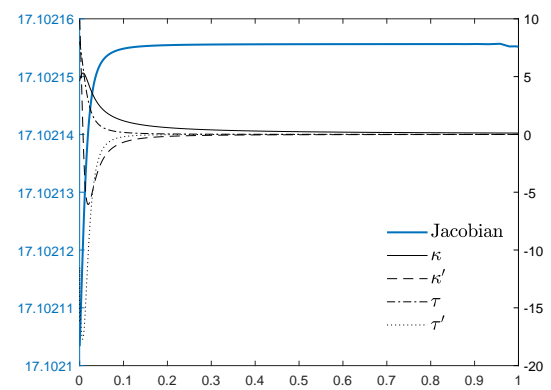

(c) Case A12.

Figure A.18: Viviani beam. $p=4, n=180$.

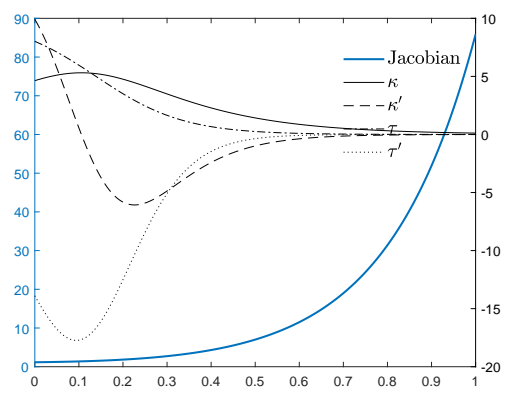

(a) Case A2.

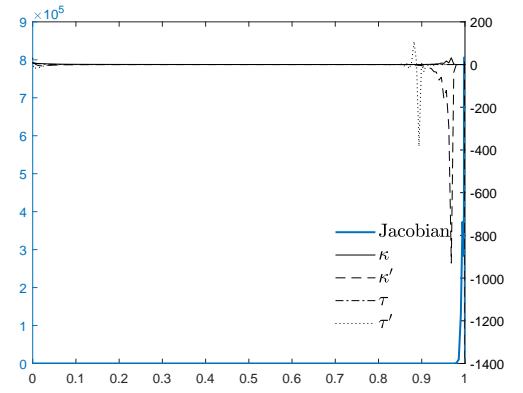

(b) Case A11.

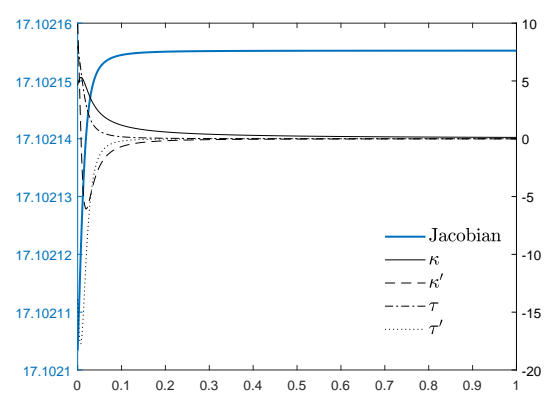

(c) Case A12.

Figure A.19: Spiral beam. $p=6, n=180$.

\section{Appendix B. Geometry approximation errors}

In this appendix we report, for each test case studied in Section 6, some figures showing the convergence of the least-square (LSQ) geometry approximation error (see Eq. (46)) for combinations A11, A2, and A12, considering both $p=4$ and $p=6$. 


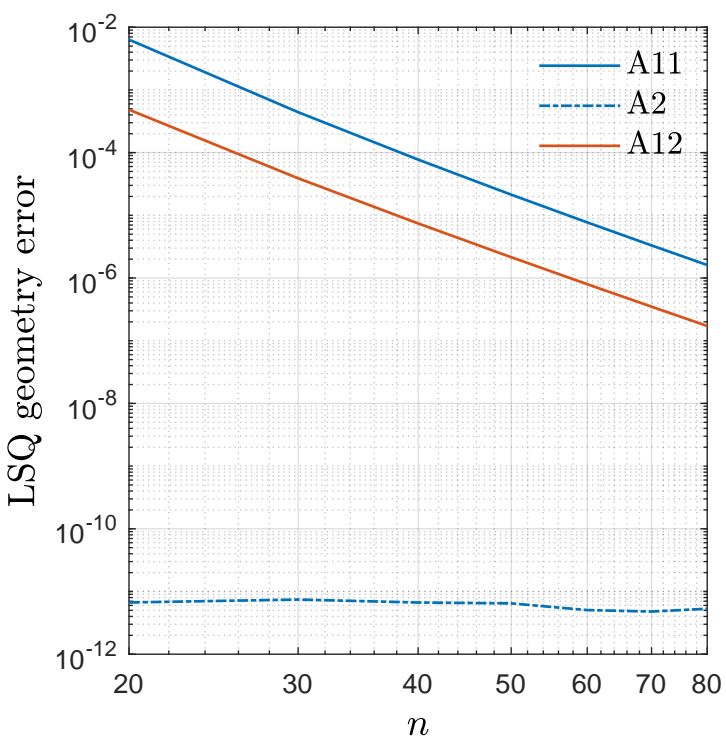

(a) $p=4$.

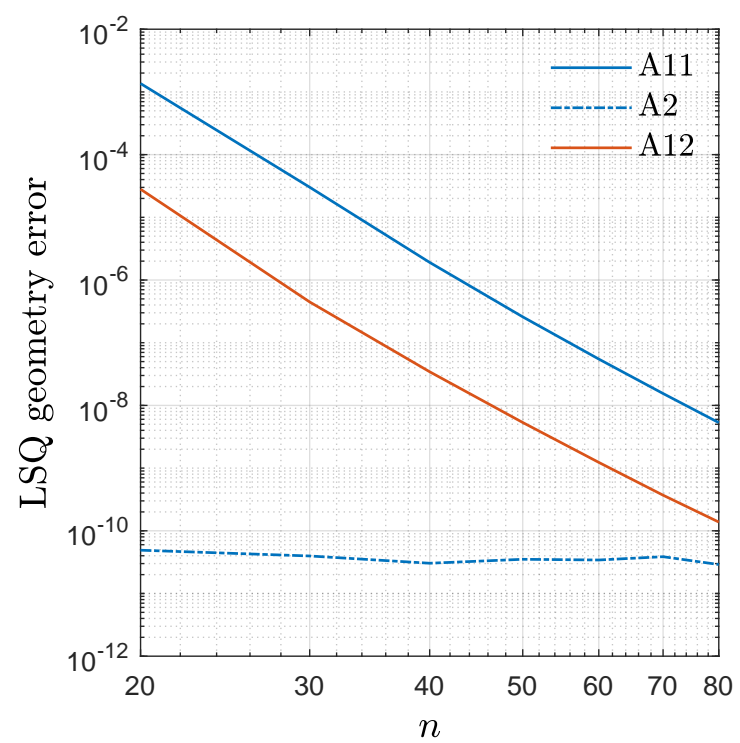

(b) $p=6$.

Figure B.20: LSQ geometry approximation error for the Tschirnhausen beam.

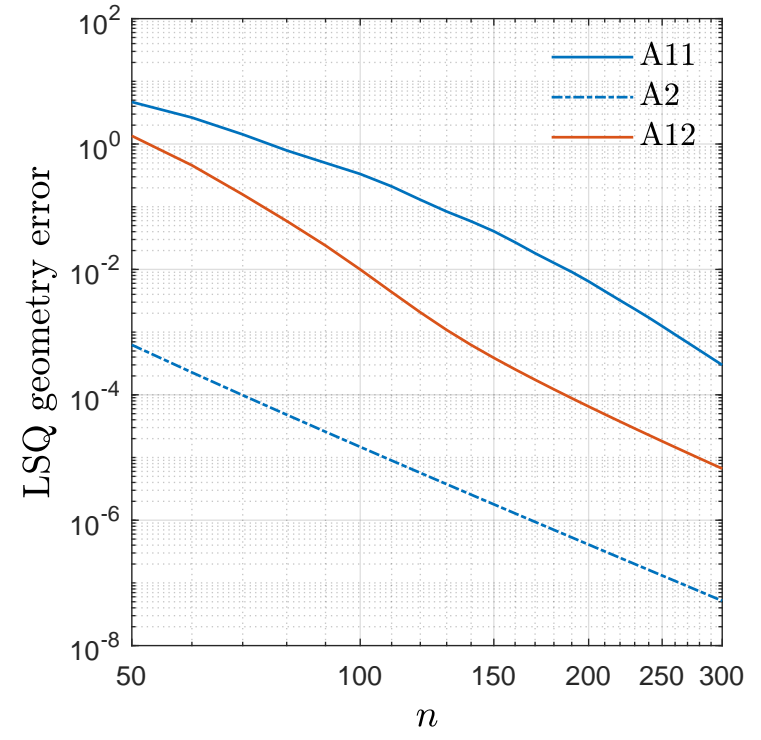

(a) $p=4$.

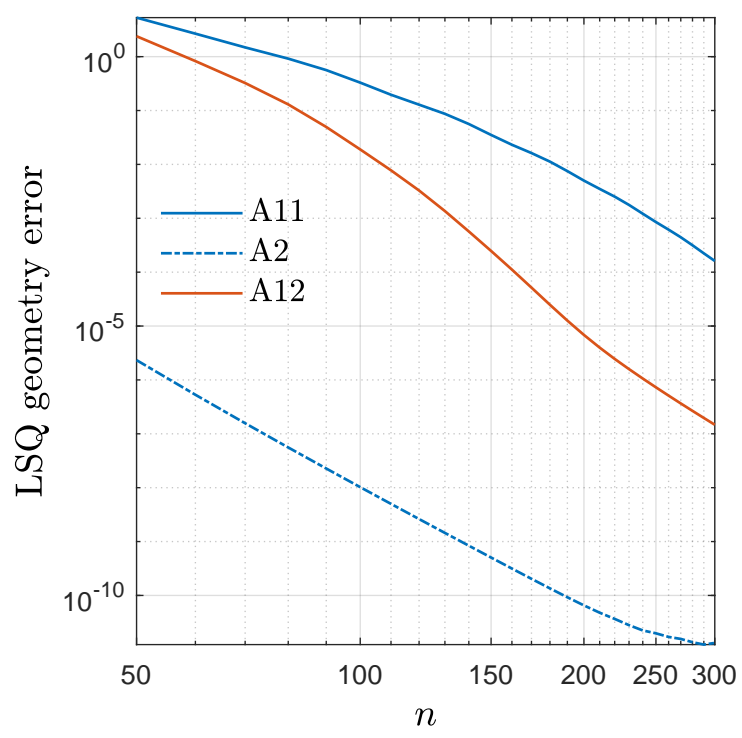

(b) $p=6$.

Figure B.21: LSQ geometry approximation error for the Lissajous beam.

\section{References}

[1] F. Auricchio, L. Beirão Da Veiga, T. J. R. Hughes, A. Reali, G. Sangalli, Isogeometric Collocation Methods, Mathematical Models and Methods in Applied Sciences 20 (11) 


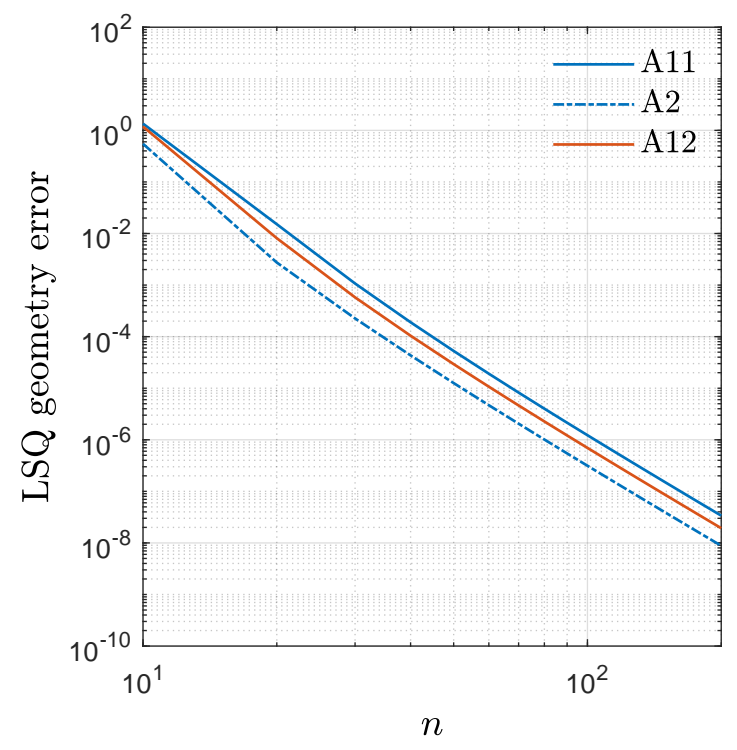

(a) $p=4$.

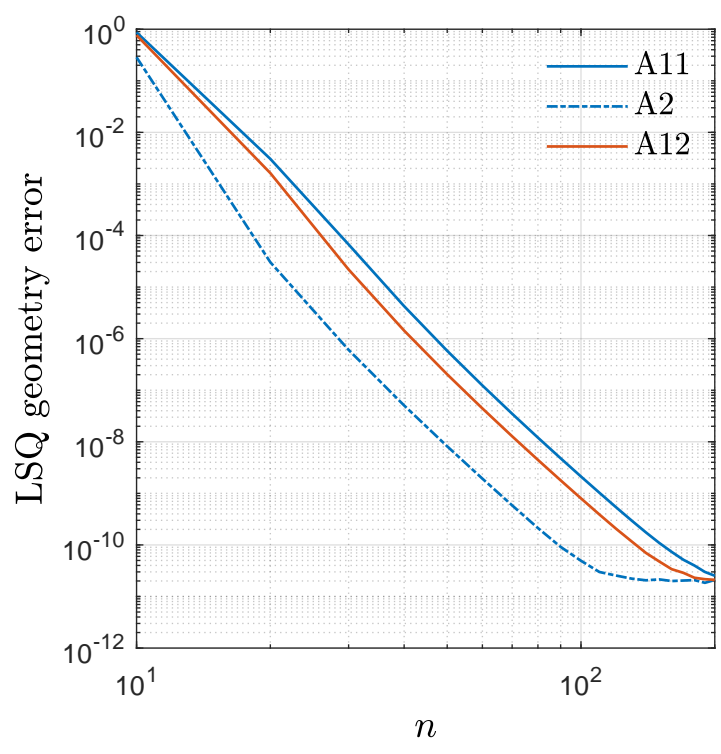

(b) $p=6$.

Figure B.22: LSQ geometry approximation error for the Viviani beam.

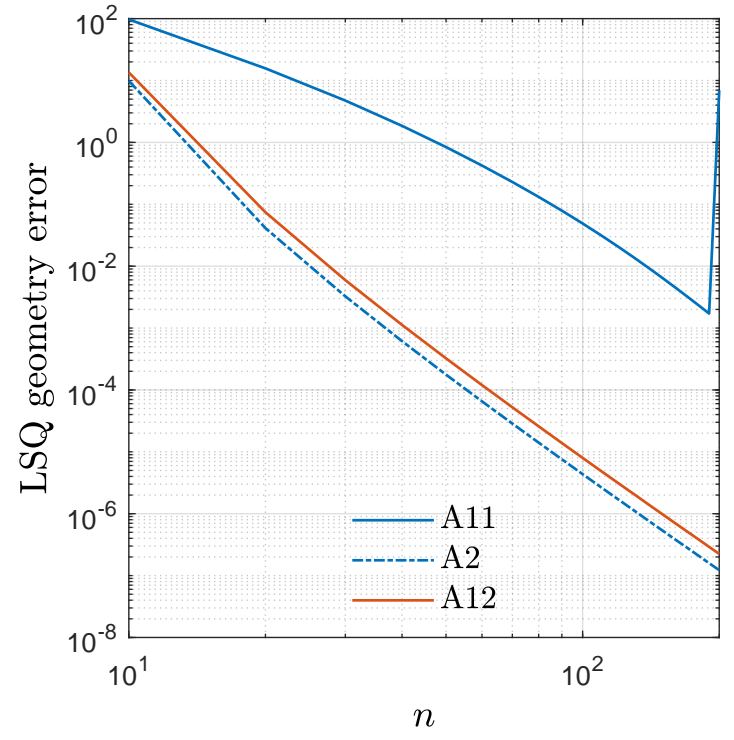

(a) $p=4$.

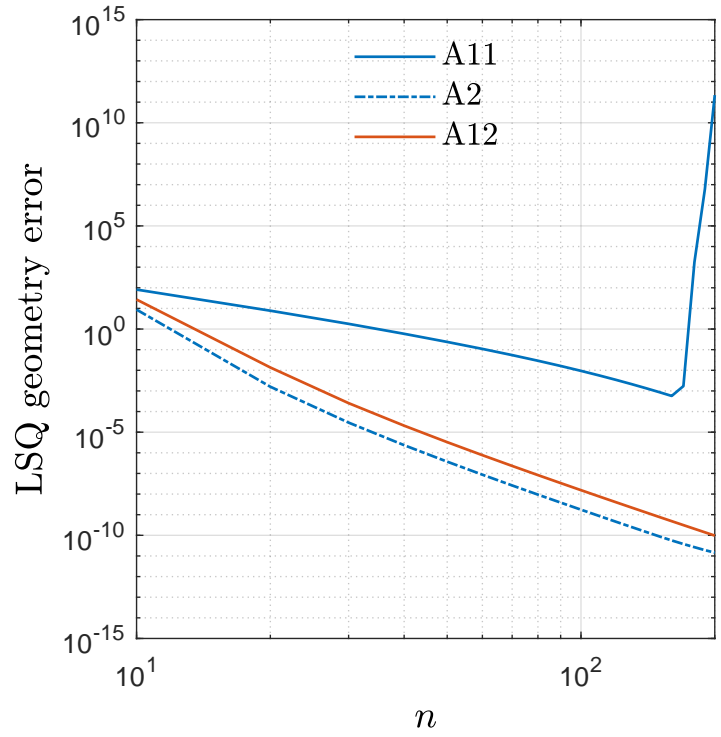

(b) $p=6$.

Figure B.23: LSQ geometry approximation error for the spiral beam.

(2010) 2075-2107.

[2] T. Hughes, J. Cottrell, Y. Bazilevs, Isogeometric analysis: CAD, finite elements, NURBS, exact geometry and mesh refinement, Computer Methods in Applied Mechan- 
ics and Engineering 194 (39-41) (2005) 4135-4195.

[3] L. Piegl, W. Tiller, The NURBS Book, Springer-Verlag, New York, NY, 1997.

[4] Y. Bazilevs, L. Beirão da Veiga, J. Cottrell, T. J. R. Hughes, G. Sangalli, Isogeometric analysis: approximation, stability and error estimates for h-refined meshes, Mathematical Models and Methods in Applied Sciences 16 (07) (2006) 1031-1090.

[5] T. J. R. Hughes, A. Reali, G. Sangalli, Duality and unified analysis of discrete approximations in structural dynamics and wave propagation: Comparison of p-method finite elements with k-method NURBS, Computer Methods in Applied Mechanics and Engineering 197 (49-50) (2008) 4104-4124.

[6] J. A. Evans, Y. Bazilevs, I. Babuška, T. J. R. Hughes, n-Widths, sup-infs, and optimality ratios for the k-version of the isogeometric finite element method, Computer Methods in Applied Mechanics and Engineering 198 (21-26) (2009) 1726-1741.

[7] L. Beirão da Veiga, A. Buffa, J. Rivas, G. Sangalli, Some estimates for h-p-k-refinement in isogeometric analysis., Numerische Mathematik 118 (2011) 271-305.

[8] J. A. Cottrell, T. J. R. Hughes, Y. Bazilevs, Isogeometric analysis: toward integration of CAD and FEA, JohnWiley \& Sons, Ltd Registered, 2009.

[9] L. Beirão da Veiga, C. Lovadina, A. Reali, Avoiding shear locking for the timoshenko beam problem via isogeometric collocation methods, Computer Methods in Applied Mechanics and Engineering 241-244 (2012) 38-51.

[10] L. De Lorenzis, P. Wriggers, G. Zavarise, A mortar formulation for 3D large deformation contact using NURBS-based isogeometric analysis and the augmented lagrangian method, Computational Mechanics 49 (2012) 1-20.

[11] L. Greco, M. Cuomo, B-Spline interpolation of Kirchhoff-Love space rods, Computer Methods in Applied Mechanics and Engineering 256 (2013) 251-269. 
[12] L. Greco, M. Cuomo, An implicit G1 multi patch B-spline interpolation for Kirchhoff-Love space rod, Computer Methods in Applied Mechanics and Engineering 269 (2014) 173-197.

[13] L. Greco, M. Cuomo, An isogeometric implicit G1 mixed finite element for Kirchhoff space rods, Computer Methods in Applied Mechanics and Engineering 298 (2016) 325349.

[14] Y. Bazilevs, V. M. Calo, T. J. R. Hughes, Y. Zhang, Isogeometric fluid-structure interaction: Theory, algorithms, and computations, Computational Mechanics 43 (2008) $3-37$.

[15] Y. Ghaffari Motlagh, H. T. Ahn, T. J. R. Hughes, V. M. Calo, Simulation of laminar and turbulent concentric pipe flows with the isogeometric variational multiscale method, Computers \& Fluids 71 (2013) 146-155.

[16] A. Hashemian, E. Lakzian, A. Ebrahimi-Fizik, On the application of isogeometric finite volume method in numerical analysis of wet-steam flow through turbine cascades, Computers \& Mathematics with Applications (2020) 1-19.

[17] A. Buffa, G. Sangalli, R. Vázquez, Isogeometric methods for computational electromagnetics: B-spline and T-spline discretizations, Journal of Computational Physics 257 (2014) $1291-1320$.

[18] D. Garcia, D. Pardo, V. M. Calo, Refined isogeometric analysis for fluid mechanics and electromagnetics, Computer Methods in Applied Mechanics and Engineering 356 (2019) $598-628$.

[19] T. J. R. Hughes, J. A. Evans, A. Reali, Finite element and NURBS approximations of eigenvalue, boundary-value, and initial-value problems, Computer Methods in Applied Mechanics and Engineering 272 (2014) 290-320.

[20] D. Wang, Q. Liang, H. Zhang, A superconvergent isogeometric formulation for eigenvalue computation of three dimensional wave equation, Computational Mechanics 57 (2016) 1037-1060. 
[21] T. Hughes, A. Reali, G. Sangalli, Efficient quadrature for NURBS-based isogeometric analysis, Computer Methods in Applied Mechanics and Engineering 199 (5-8) (2010) $301-313$.

[22] F. Auricchio, F. Calabrò, T. Hughes, A. Reali, G. Sangalli, A simple algorithm for obtaining nearly optimal quadrature rules for NURBS-based isogeometric analysis, Computer Methods in Applied Mechanics and Engineering 249-252 (2012) 15-27.

[23] C. Adam, T. J. R. Hughes, S. Bouabdallah, M. Zarroug, H. Maitournam, Selective and reduced numerical integrations for NURBS-based isogeometric analysis, Computer Methods in Applied Mechanics and Engineering 284 (2015) 732-761.

[24] P. Antolin, A. Buffa, F. Calabrò, M. Martinelli, G. Sangalli, Efficient matrix computation for tensor-product isogeometric analysis: The use of sum factorization, Computer Methods in Applied Mechanics and Engineering 285 (2015) 817-828.

[25] F. Calabrò, G. Sangalli, M. Tani, Fast formation of isogeometric Galerkin matrices by weighted quadrature, Computer Methods in Applied Mechanics and Engineering 316 (2017) 606-622.

[26] F. Fahrendorf, L. De Lorenzis, H. Gomez, Reduced integration at superconvergent points in isogeometric analysis, Computer Methods in Applied Mechanics and Engineering 328 (2018) 390-410.

[27] G. Sangalli, M. Tani, Matrix-free weighted quadrature for a computationally efficient isogeometric k-method, Computer Methods in Applied Mechanics and Engineering 338 (2018) 117-133.

[28] R. R. Hiemstra, G. Sangalli, M. Tani, F. Calabrò, T. J. Hughes, Fast formation and assembly of finite element matrices with application to isogeometric linear elasticity, Computer Methods in Applied Mechanics and Engineering 355 (2019) 234-260.

[29] D. Schillinger, J. Evans, A. Reali, M. Scott, T. J. R. Hughes, Isogeometric collocation: Cost comparison with Galerkin methods and extension to adaptive hierarchical NURBS 
discretizations, Computer Methods in Applied Mechanics and Engineering 267 (2013) $170-232$.

[30] F. Auricchio, L. Beirão da Veiga, T. J. R. Hughes, A. Reali, G. Sangalli, Isogeometric collocation for elastostatics and explicit dynamics, Computer Methods in Applied Mechanics and Engineering 249-252 (2012) 2-14.

[31] H. Gomez, A. Reali, G. Sangalli, Accurate, efficient, and (iso)geometrically flexible collocation methods for phase-field models., Journal for Computational Physics 262 (2014) 153-171.

[32] P. Fedeli, A. Frangi, F. Auricchio, A. Reali, Phase-field modeling for polarization evolution in ferroelectric materials via an isogeometric collocation method, Computer Methods in Applied Mechanics and Engineering 351 (2019) 789-807.

[33] L. De Lorenzis, J. Evans, T. Hughes, A. Reali, Isogeometric collocation: Neumann boundary conditions and contact, Computer Methods in Applied Mechanics and Engineering 284 (2015) 21-54.

[34] R. Kruse, N. Nguyen-Thanh, L. De Lorenzis, T. Hughes, Isogeometric collocation for large deformation elasticity and frictional contact problems, Computer Methods in Applied Mechanics and Engineering 296 (2015) 73-112.

[35] H. Gomez, L. De Lorenzis, The variational collocation method, Computer Methods in Applied Mechanics and Engineering 309 (2016) 152-181.

[36] L. Beirão da Veiga, C. Lovadina, a. Reali, Avoiding shear locking for the Timoshenko beam problem via isogeometric collocation methods, Computer Methods in Applied Mechanics and Engineering 241-244 (2012) 38-51.

[37] F. Auricchio, L. Beirão da Veiga, J. Kiendl, C. Lovadina, a. Reali, Locking-free isogeometric collocation methods for spatial Timoshenko rods, Computer Methods in Applied Mechanics and Engineering 263 (2013) 113-126. 
[38] J. Kiendl, F. Auricchio, T. Hughes, A. Reali, Single-variable formulations and isogeometric discretizations for shear deformable beams, Computer Methods in Applied Mechanics and Engineering 284 (2015) 988-1004.

[39] J. Kiendl, F. Auricchio, A. Reali, A displacement-free formulation for the Timoshenko beam problem and a corresponding isogeometric collocation approach, Meccanica (2017) $1-11$.

[40] G. Balduzzi, S. Morganti, F. Auricchio, Non-prismatic Timoshenko-like beam model: Numerical solution via isogeometric collocation, Computers \& Mathematics with Applications 74 (7) (2017) 1531-1541.

[41] A. Reali, H. Gomez, An isogeometric collocation approach for Bernoulli-Euler beams and Kirchhoff plates, Computer Methods in Applied Mechanics and Engineering 284 (2015) 623-636.

[42] J. Kiendl, F. Auricchio, L. Beirão da Veiga, C. Lovadina, A. Reali, Isogeometric collocation methods for the Reissner-Mindlin plate problem, Computer Methods in Applied Mechanics and Engineering 284 (2015) 489-507.

[43] J. Kiendl, E. Marino, L. De Lorenzis, Isogeometric collocation for the Reissner-Mindlin shell problem, Computer Methods in Applied Mechanics and Engineering 325 (2017) 645-665.

[44] F. Maurin, F. Greco, L. Coox, D. Vandepitte, W. Desmet, Isogeometric collocation for Kirchhoff-Love plates and shells, Computer Methods in Applied Mechanics and Engineering 329 (2018) 396-420.

[45] A. Patton, J. E. Dufour, P. Antolin, A. Reali, Fast and accurate elastic analysis of laminated composite plates via isogeometric collocation and an equilibrium-based stress recovery approach, Composite Structures 225 (oct 2019).

[46] F. Maurin, F. Greco, S. Dedoncker, W. Desmet, Isogeometric analysis for nonlinear planar Kirchhoff rods: Weighted residual formulation and collocation of the strong form, Computer Methods in Applied Mechanics and Engineering (2018). 
[47] J. A. Evans, R. R. Hiemstra, T. J. R. Hughes, A. Reali, Explicit higher-order accurate isogeometric collocation methods for structural dynamics, Computer Methods in Applied Mechanics and Engineering 338 (2018) 208-240.

[48] E. Marino, Isogeometric collocation for three-dimensional geometrically exact sheardeformable beams, Computer Methods in Applied Mechanics and Engineering 307 (2016) 383-410.

[49] O. Weeger, S.-K. Yeung, M. L. Dunn, Isogeometric collocation methods for Cosserat rods and rod structures, Computer Methods in Applied Mechanics and Engineering 316 (2017) 100-122.

[50] E. Marino, Locking-free isogeometric collocation formulation for three-dimensional geometrically exact shear-deformable beams with arbitrary initial curvature, Computer Methods in Applied Mechanics and Engineering 324 (2017) 546-572.

[51] O. Weeger, B. Narayanan, M. L. Dunn, Isogeometric collocation for nonlinear dynamic analysis of Cosserat rods with frictional contact, Nonlinear Dynamics (2017) 1-15.

[52] E. Marino, J. Kiendl, L. De Lorenzis, Explicit isogeometric collocation for the dynamics of three-dimensional beams undergoing finite motions, Computer Methods in Applied Mechanics and Engineering 343 (2019) 530-549.

[53] E. Marino, J. Kiendl, L. De Lorenzis, Isogeometric collocation for implicit dynamics of three-dimensional beams undergoing finite motions, Computer Methods in Applied Mechanics and Engineering 356 (2019) 548-570.

[54] E. Cohen, T. Martin, R. M. Kirby, T. Lyche, R. F. Riesenfeld, Analysis-aware modeling: Understanding quality considerations in modeling for isogeometric analysis, Computer Methods in Applied Mechanics and Engineering 199 (2010) 334-356.

[55] G. Xu, B. Mourrain, R. Duvigneau, A. Galligo, Optimal analysis-aware parameterization of computational domain in 3D isogeometric analysis, Computer-Aided Design 45 (2013) 812-821. 
[56] G. Xu, M. Li, B. Mourrain, T. Rabczuk, J. Xu, S. P. Bordas, Constructing IGAsuitable planar parameterization from complex CAD boundary by domain partition and global/local optimization, Computer Methods in Applied Mechanics and Engineering 328 (2018) 175-200.

[57] H. Casquero, L. Liu, Y. Zhang, A. Reali, H. Gomez, Isogeometric collocation using analysis-suitable T-splines of arbitrary degree, Computer Methods in Applied Mechanics and Engineering 301 (2016) 164-186.

[58] S. Lipton, J. A. Evans, Y. Bazilevs, T. Elguedj, T. J. R. Hughes, Robustness of isogeometric structural discretizations under severe mesh distortion, Computer Methods in Applied Mechanics and Engineering 199 (2010) 357-373.

[59] S. F. Hosseini, A. Hashemian, A. Reali, On the application of curve reparameterization in isogeometric vibration analysis of free-from curved beams, Computers \& Structures 209 (2018) 117-129.

[60] A. Hashemian, S. F. Hosseini, S. N. Nabavi, Kinematically smoothing trajectories by NURBS reparameterization - an innovative approach, Advanced Robotics 31 (2017) 1296-1312.

[61] L. Biagiotti, C. Melchiorri, Trajectory Planning for Automatic Machines and Robots, Springer-Verlag, Berlin Heidelberg, 2008.

[62] Q. Zhang, S. Li, J. Guo, Smooth time-optimal tool trajectory generation for CNC manufacturing systems, Journal of Manufacturing Systems 31 (2012) 280-287.

[63] J. Zhao, Q. Zou, L. Li, B. Zhou, Tool path planning based on conformal parameterization for meshes, Chinese Journal of Aeronautics 28 (2015) 1555-1563.

[64] R. Kolman, J. Plešek, M. Okrouhlík, Complex wavenumber fourier analysis of the Bspline based finite element method, Wave Motion 51 (2014) 348-359.

[65] S. F. Hosseini, B. Moetakef-Imani, S. Hadidi-Moud, B. Hassani, The effect of parameterization on isogeometric analysis of free-form curved beams, Acta Mechanica 227 (2016) 1983-1998. 
[66] J. A. Cottrell, A. Reali, Y. Bazilevs, T. J. R. Hughes, Isogeometric analysis of structural vibrations, Computer Methods in Applied Mechanics and Engineering 195 (2006) 52575296.

[67] S. Hosseini, B. Moetakef-Imani, S. Hadidi-moud, B. Hassani, Pre-bent shape design of full free-form curved beams using isogeometric method and semi-analytical sensitivity analysis, Structural and Multidisciplinary Optimization 58 (2018) 2621-2633.

[68] S. Shojaee, N. Valizadeh, E. Izadpanah, T. Bui, T.-V. Vu, Free vibration and buckling analysis of laminated composite plates using the NURBS-based isogeometric finite element method, Composite Structures 94 (2012) 1677-1693.

[69] D. Wang, W. Liu, H. Zhang, Superconvergent isogeometric free vibration analysis of Euler-Bernoulli beams and kirchhoff plates with new higher order mass matrices, Computer Methods in Applied Mechanics and Engineering 286 (2015) 230-267.

[70] S. F. Hosseini, A. Hashemian, A. Reali, Studies on knot placement techniques for the geometry construction and the accurate simulation of isogeometric spatial curved beams, Computer Methods in Applied Mechanics and Engineering (2020) 112705.

[71] M. P. Do Carmo, Differential geometry of curves and surfaces, Prentice-Hall, Inc., 1976.

[72] B. Tabarrok, M. Farshad, H. Yi, Finite element formulation of spatially curved and twisted rods, Computer Methods in Applied Mechanics and Engineering 70 (3) (1988) $275-299$.

[73] K. Arunakirinathar, B. D. Reddy, Mixed finite element methods for elastic rods of arbitrary geometry, Numerische Mathematik 64 (1993) 13-43.

[74] G. Zhang, R. Alberdi, K. Khandelwal, Analysis of three-dimensional curved beams using isogeometric approach, Engineering Structures 117 (2016) 560-574.

[75] X. Zhang, Y. Xia, Q. Hu, P. Hu, Efficient isogeometric formulation for vibration analysis of complex spatial beam structures, European Journal of Mechanics, A/Solids 66 (2017) $212-231$. 
[76] R. L. Bishop, There is More than One Way to Frame a Curve, The American Mathematical Monthly 82 (3) (1975) 246.

[77] O. Weeger, B. Narayanan, M. L. Dunn, Isogeometric shape optimization of nonlinear, curved 3D beams and beam structures, Computer Methods in Applied Mechanics and Engineering 345 (2019) 26-51.

[78] B. M. Imani, S. A. Hashemian, NURBS-based profile reconstruction using constrained fitting techniques, Journal of Mechanics 28 (2012) 407-412.

[79] A. Hashemian, S. F. Hosseini, An integrated fitting and fairing approach for object reconstruction using smooth NURBS curves and surfaces, Computers \& Mathematics with Applications 76 (2018) 1555-1575.

[80] L. Piegl, W. Tiller, Surface approximation to scanned data, The Visual Computer 16 (2000) 386-395.

[81] H. Park, B-spline surface fitting based on adaptive knot placement using dominant columns, Computer-Aided Design 43 (2011) 258-264.

[82] F. Pérez-Arribas, R. Pérez-Fernández, A B-spline design model for propeller blades, Advances in Engineering Software 118 (2018) 35-44.

[83] C. Anitescu, Y. Jia, Y. J. Zhang, T. Rabczuk, An isogeometric collocation method using superconvergent points, Computer Methods in Applied Mechanics and Engineering 284 (2015) 1073-1097.

[84] M. Montardini, G. Sangalli, L. Tamellini, Optimal-order isogeometric collocation at Galerkin superconvergent points, Computer Methods in Applied Mechanics and Engineering 316 (2017) 741-757.

[85] Y. Jia, C. Anitescu, Y. J. Zhang, T. Rabczuk, An adaptive isogeometric analysis collocation method with a recovery-based error estimator, Computer Methods in Applied Mechanics and Engineering 345 (2019) 52-74. 
[86] A.-T. Luu, N.-I. Kim, J. Lee, Isogeometric vibration analysis of free-form timoshenko curved beams, Meccanica 50 (2015) 169-187.

[87] A. Hashemian, S. F. Hosseini, Nonlinear bifurcation analysis of statically loaded freeform curved beams using isogeometric framework and pseudo-arclength continuation, International Journal of Non-Linear Mechanics 113 (2019) 1-16.

[88] L. R. Costa, E. Marchetti, Mathematical and historical investigation on domes and vaults, in: R. Weber, M. A. Amann (Eds.), Aesthetics and architectural composition: Proceedings of the Dresden International Symposium of Architecture 2004, Pro Literatur, Mammendorf, Germany, 2005, pp. 73-80. 